\title{
C AND N ABUNDANCES IN STARS AT THE BASE OF THE RED GIANT BRANCH IN M15 ${ }^{1}$

\author{
Judith G. Cohen, ${ }^{2}$ Michael M. Briley, ${ }^{3}$ and Peter B. Stetson ${ }^{4,5,6}$ \\ Received 2005 January 31; accepted 2005 May 11
}

\begin{abstract}
We present an analysis of a large sample of moderate-resolution Keck Low Resolution Imaging Spectrometer spectra of subgiants and stars at the base of the red giant branch (RGB) in the Galactic globular cluster (GC) M15 (NGC 7078), most within the range $16.5<V<19.5\left(1.2<M_{V}<4.2\right)$, with the goal of deriving $\mathrm{C}$ abundances (from the $\mathrm{G}$ band of $\mathrm{CH}$ ) and $\mathrm{N}$ abundances (from the $\mathrm{NH}$ band at $3360 \AA$ ). Star-to-star stochastic variations with significant range in both [C/Fe] and [N/Fe] are found at all luminosities extending to the subgiants at $M_{V} \sim+3$. The $\mathrm{C}$ and $\mathrm{N}$ abundances appear anticorrelated, as would be expected from the CN-cycle processing of stellar material. Yet these M15 stars are considerably fainter than the RGB bump, the point at which deep mixing is believed to set in. On this basis, while the observed abundance pattern is consistent with proton-capture nucleosynthesis, we infer that the site of the reactions is likely not within the present sample. The range of variation of the $\mathrm{N}$ abundances is very large, and the sum of $\mathrm{C}+\mathrm{N}$ increases as $\mathrm{C}$ decreases. To reproduce this requires the incorporation not only of $\mathrm{CN}$ but also of ON-processed material. Combining our work with that of Trefzger and coworkers for the brighter giants in M15, we find strong evidence for additional depletion of C among the most luminous giants. This presumably represents the first dredge-up (with enhanced deep mixing) expected for such luminous cluster RGB stars in the course of normal stellar evolution as they cross the RGB bump.

We compare the behavior of these patterns for $\mathrm{C}$ and $\mathrm{N}$ in GCs covering a wide range of metallicity and current mass. While all clusters studied show strong anticorrelated variations of $\mathrm{C}$ and $\mathrm{N}$ at all luminosities probed, the metalrich clusters (M71, 47 Tuc, and M5) do not show evidence for the first dredge-up among their most luminous giants, while the metal-poor ones (M13, M92, and M15, plus M5) do. Conversely, the metal-poor clusters do not show evidence for the bimodality in $\mathrm{CH}$ and $\mathrm{CN}$ line strengths seen in the metal-rich clusters. The collected data on $\mathrm{C}$ and $\mathrm{N}$ abundances in low-luminosity GC stars cannot be explained by the commonly invoked models for the chemical evolution of GC stars; in particular, "pollution" of existing low-mass stars by ejecta from intermediate-mass asymptotic giant branch (AGB) stars can be ruled out. Pollution of cluster gas by such stars prior to the formation of the lower mass stars we observe today can also be ruled out unless current models of nucleosynthesis and dredge-up into the surface layers of AGB stars are flawed; such models agree qualitatively but disagree quantitatively with our data. We are forced to assume that there was an extended period of star formation in GCs, and that a previous generation of more massive stars evolved, ejected mass, and polluted the GC gas with light elements; the low-mass stars we see today formed afterward. A tentative scenario is developed involving an initial phase of star formation heavily biased toward high-mass stars, with subsequent formation of intermediate-mass, then low-mass stars.
\end{abstract}

Key words: globular clusters: general — globular clusters: individual (M15) — stars: abundances — stars: evolution

Online material: machine-readable tables

\section{INTRODUCTION}

By virtue of their large populations of coeval stars, the Galactic globular clusters ( $\mathrm{GCs}$ ) present us with a unique laboratory for the study of the evolution of low-mass stars. The combination of their extreme ages, compositions, and dynamics also allows us a glimpse at the early history of the Milky Way and the

\footnotetext{
${ }^{1}$ Based on observations obtained at the W. M. Keck Observatory, which is operated jointly by the California Institute of Technology, the University of California, and the National Aeronautics and Space Administration.

2 Palomar Observatory, California Institute of Technology, Mail Stop 105-24, Pasadena, CA 91125; jlc@astro.caltech.edu.

${ }^{3}$ Department of Physics and Astronomy, University of Wisconsin-Oshkosh, 800 Algoma Boulevard, Oshkosh, WI 54901; currently on assignment to the National Science Foundation, 4201 Wilson Boulevard, Arlington, VA 22230; mike@ maxwell.phys.uwosh.edu. Any opinions, findings, conclusions, and recommendations expressed in this material are those of the author and do not necessarily reflect the views of the National Science Foundation.

${ }^{4}$ Dominion Astrophysical Observatory, 5071 West Saanich Road, Victoria, BC V9E 2E7, Canada; peter.stetson@hia.nrc.ca.

${ }^{5}$ Guest User, Canadian Astronomy Data Centre, which is operated by the Herzberg Institute of Astrophysics, National Research Council of Canada.

${ }^{6}$ Guest Investigator, UK Astronomy Data Centre.
}

processes operating during its formation. These aspects become even more significant in the context of the star-to-star light-element inhomogeneities found among red giants in every cluster studied to date. The large differences in the surface abundances of $\mathrm{C}, \mathrm{N}, \mathrm{O}$, and often $\mathrm{Na}, \mathrm{Mg}$, and $\mathrm{Al}$ have defied a comprehensive explanation in the three decades since their discovery.

Proposed origins of the inhomogeneities typically break down into two scenarios: (1) As C, N, O, Na, $\mathrm{Mg}$, and $\mathrm{Al}$ are related to proton-capture processes at $\mathrm{CN}$ - and $\mathrm{CNO}$-burning temperatures, material cycled through a region in the upper layers of the H-burning shell in evolving cluster giants may be brought to the surface with accompanying changes in composition; see Sweigart \& Mengel (1979) and Charbonnel (1994) for an introduction to the relevant theory of mixing. There is ample observational evidence that deep mixing takes place during the red giant branch (RGB) ascent of metal-poor cluster stars (see the reviews of Kraft [1994], Pinsonneault [1997], and Gratton et al. [2004] and references therein). (2) It has also become apparent that at least some component of these abundance variations must be in place before some cluster stars reach the giant branch. Spectroscopic observations of main-sequence turnoff stars in 47 Tuc (beginning with 
Hesser [1978]; see Cannon et al. [1998] and Briley et al. [2004b] and references therein) demonstrate this. Our own work with large samples of low-luminosity stars in M71 (Cohen 1999; Briley \& Cohen 2001; Ramírez \& Cohen 2002) and M13 (Briley et al. 2002, 2004a; Cohen \& Meléndez 2005) have shown variations in $\mathrm{CN}$ - and $\mathrm{CH}-$ band and $\mathrm{O}$ and $\mathrm{Na}$ line strengths among subgiants and main-sequence stars consistent with patterns found among the evolved giants of these clusters. The low-mass cluster stars we observe are, however, incapable of both deep dredge-up and significant $\mathrm{CNO}$ nucleosynthesis while on the main sequence. Hence, the early cluster material must have been at least partially inhomogeneous in these elements, or some form of modification of these elements took place within the cluster. Suggested culprits include mass loss from intermediate-mass asymptotic giant branch (AGB) stars and supernova ejecta. Cannon et al. (1998) present an excellent discussion of these possibilities.

Thus, the observed light-element inhomogeneities imply that there is some aspect of the structure of the evolving cluster giants that remains poorly understood (the deep-mixing mechanism), that the early protoclusters may have been far less homogeneous, that intermediate-mass stars may have played a greater role in setting the composition of the present-day lowmass stars than previously thought, etc. It is this set of issues that we explore in the present series of papers. In our earlier work we studied the $\mathrm{C}$ and $\mathrm{N}$ abundances for large samples of stars in the GCs M71 (Cohen 1999; Briley \& Cohen 2001), M5 (Cohen et al. 2002), and M13 (Briley et al. 2002, 2004a) (collectively denoted GC-CN). We consider here the extremely metal-poor GC M15. In this range of metallicity and luminosity (i.e., $T_{\text {eff }}$ ), the $\mathrm{CN}$ bands at 3880 and $4220 \AA$ in the spectra of the M15 stars are too weak to be useful, so we rely on the $\mathrm{CH}$ and $\mathrm{NH}$ band strengths to derive $\mathrm{C}$ and $\mathrm{N}$ abundances for a large sample of stars in M15. We adopt values from the online database of Harris (1996) for the apparent distance modulus of M15 at $V$ of 15.31 mag with a reddening of $E(B-V)=0.09 \mathrm{mag}$, supported by analysis of deep Hubble Space Telescope (HST) photometry by Recio-Blanco et al. (2005). We adopt the metallicity $[\mathrm{Fe} / \mathrm{H}]=-2.2 \mathrm{dex}$, also from Harris (1996); Sneden et al. (1997), in a high dispersion abundance analysis of a large sample of stars on the upper giant branch of M15, found a somewhat lower value.

We describe the sample in $\S \S 2$ and 3. We outline our measurement of the molecular band indices and their interpretation in $\S 4$. With an assumption about the $\mathrm{O}$ abundance, these are converted into $\mathrm{C}$ and $\mathrm{N}$ abundances in $\S 5$, from which we find an anticorrelation between $\mathrm{C}$ and $\mathrm{N}$ in $\S 6$. A consideration of the need for ON burning follows in $\S 7$. A discussion of our results together with a comparison with the trends seen among the red giants in M15 with our earlier work, which now covers four GCs spanning a wide range of metallicity, combined with data from the literature, is given in $\S 8$, while $\S 9$ discusses the implications of our results for the mechanism producing the $\mathrm{C}$ and $\mathrm{N}$ differences. Inferences we can draw from this for the formation and early chemical history of GCs are given in $\S 10$. A brief summary $(\S 11)$ concludes the paper.

\section{PHOTOMETRIC DATABASES}

The optical photometry of M15 employed here was carried out as part of a larger program to provide homogeneous photometry for star clusters and nearby resolved galaxies (Stetson 2000). The general characteristics of the photometric database are described in detail in Cohen et al. (2002) for the case of M5; the case of M15 is similar. At the time the present photometry for M15 was derived, the corpus of M15 images in Stetson's database included some 307 images in $B, 340$ images in $V$, and 181 images in $I$. The images did not all cover the same region of sky, of course, and any given star fell within no more than $235 \mathrm{~B}$ images, $239 \mathrm{~V}$ images, or $179 \mathrm{I}$ images. A network of local standards supported appropriate transformations for those images taken under nonphotometric conditions. In our experience, photometry from data sets such as those employed here typically displays an external accuracy of order 0.02 mag per observation; this level of observation-to-observation scatter is probably dominated by temporal and spatial fluctuations in the instantaneous atmospheric extinction and probably also by the difficulty of obtaining truly appropriate flat-field corrections in the presence of such effects as scattered light, ghosts, fringing, and spectral mismatch between the flat-field illumination and the astronomical scene. The fundamental system is that of Landolt (1992).

The absolute astrometry of our catalog is based on the United States Naval Observatory A2.0 Guide Star Catalog I (USNOGSC; Monet et al. 1998), access to which is obtained by P. B. S. through the services of the Canadian Astronomy Data Centre. Throughout the region of our field that is well populated by USNOGSC stars (including essentially all of the stars in our present spectroscopic sample), we expect systematic errors of our right ascensions and declinations on the system of the USNOGSC to be $<0$.' 1 . Individual random errors in our coordinate measurements are probably not much better than 0.02 on a star-by-star basis, the errors becoming somewhat worse than this for the fainter and more crowded stars in our photometricastrometric sample.

The alignment images for the slit masks used with the Low Resolution Imaging Spectrometer (LRIS; Oke et al. 1995) were taken with $V$ or $I$ filters. Although these exposures were short (typically $1 \mathrm{~s}$ ), they were used to determine $V$ magnitudes for the faintest stars, particularly those that were somewhat crowded, in our sample in M15.

To broaden the wavelength range of our photometry, we attempted to obtain infrared colors from the Two Micron All Sky Survey (2MASS) database (Skrutskie et al. 1997; Cutri et al. 2003) for the stars in our sample. However, many of them are too faint to be included therein. Thus, in 2004 September we observed the fields of our M15 sample with the Wide Field Infrared Camera (WIRC; Wilson et al. 2003) at the $5 \mathrm{~m}$ Hale Telescope for the purpose of establishing reliable $J, K$ magnitudes for the fainter stars in our sample. The 2MASS colors of nearby, isolated, somewhat brighter stars were used to calibrate our WIRC photometry. Total integrations of 10-30 minutes for each of the two filters in each of the two fields were obtained. These images were reduced using Figaro (Shortridge 1993) and DAOPHOT (Stetson 1987).

Most other recent photometric studies of M15 (Buonanno et al. 1985; DaCosta \& Armandroff 1990) do not reach as faint as the bulk of our sample. The deep $B, V$ color-magnitude diagram (CMD) study of Durrell \& Harris (1993), which focuses on the age of the cluster, its distance, and its luminosity function along the main sequence, does not cover the full sample of our stars. Stars are identified in this paper by a name derived from their J2000.0 coordinates, so that star C12345_5432 has coordinates R.A. $=21^{\mathrm{h}} 12^{\mathrm{m}} 34^{\mathrm{s}} .5$, decl. $=+12^{\circ} 54^{\prime} 32^{\prime \prime}$.

\section{SPECTROSCOPIC OBSERVATIONS}

The initial sample of stars consisted of those from the photometric database located more than $180^{\prime \prime}$ from the center of M15 (to avoid crowding) with $16.5<V<18.5$ and with $B-V$ within 0.06 mag of the cluster locus, which we take as $B-V=$ $0.73-0.062(V-16.5) \mathrm{mag}$. The main-sequence turnoff of 
M15 is at $V \sim 19.2 \mathrm{mag}$, so these stars include subgiants, as well as low-luminosity giants near the base of the RGB. (A preliminary version of the photometric catalog described in $\S 2$ was used for this purpose.) From this list, two slit masks containing about 25 slitlets each were designed using J. G. C.'s software. The center of the first field was roughly 3.'2 east and 0.7 north of the center of M15, while the center of the second field was located roughly 2.'8 west and 2.'9 south of the cluster center.

These slit masks were used with LRIS at the Keck Observatory in 2003 June. Three 1200 s exposures were obtained for the second slit mask and two $1200 \mathrm{~s}$ exposures for the first slit mask. The air mass was less than 1.06 for all exposures, so differential refraction did not play a role even at $3200 \AA$. The exposures were dithered by moving the stars along the length of the slitlets by $2^{\prime \prime}$ between each exposure. Because of the crowded fields, there was often more than one suitably bright object in each slitlet. The width of the slitlets was 0.8 , narrower than normal to enhance the spectral resolution. LRIS-B (McCarthy et al. 1998) was used with a 400 line grism, giving a dispersion of $1.0 \AA$ pixel $^{-1}$ (4.0 $\AA$ resolution for a $0 . \prime 8$ wide slit). This gave good coverage of the region from 3000 to $5000 \AA$, including the key NH band at $3360 \AA$ and the $\mathrm{G}$ band of $\mathrm{CH}$ at $4300 \AA$. The $\mathrm{CN}$ bands at 3880 and $4200 \AA$, while included within the spectral range covered, are too weak to be measured with precision for most of these low-luminosity, very metal-poor stars; they were not used at all. The red side of LRIS was configured to use a 1200 grooves $\mathrm{mm}^{-1}$ grating centered at $\mathrm{H} \alpha$ with the intention of providing higher accuracy radial velocities. The dispersion was then $0.64 \AA$ pixel $^{-1}\left(29 \mathrm{~km} \mathrm{~s}^{-1}\right.$ pixel $\left.^{-1}\right)$ or $1.9 \AA$ per spectral resolution element. Figaro (Shortridge 1993) scripts were used for the data reduction.

The original detector of LRIS-B was upgraded to a new one with much higher UV sensitivity prior to these observations. This was crucial to the success of our effort. However, there were some unexpectedly severe reflection problems in our bluechannel spectra. These were perhaps exacerbated by the many bright stars in the field. The reflections were nondispersed, aligned along the slit, and several times the height of a stellar image. They were removed partially by sky subtraction, but the resulting spectra had to be hand-checked, with additional corrections applied as necessary. This was done for each individual exposure, then the resulting spectra for each star were summed. In addition, the LRIS-B images were not flattened, as there is no suitably bright featureless UV calibration lamp at the Keck Observatory. The pixel-to-pixel variation is small in these detectors, and each spectrum is the sum of several exposures that fell in different locations on the detector array. Furthermore, the projected image size of a point source along the slit has a FWHM of 3-4 pixels, so many pixels are sampled in forming the final one-dimensional spectrum for each star. We therefore are confident that the lack of flat-fielding does not introduce spurious small-scale features.

The final spectrum summed over the three $1200 \mathrm{~s}$ exposures of an M15 star in our sample with $V=18.0$ mag has roughly 9000 detected electrons per spectral pixel $(1.0 \AA)$ in the region of the $\mathrm{G}$ band of $\mathrm{CH}$ and roughly 1100 detected electrons per spectral pixel in the blue continuum bandpass for the NH feature. For a $V=18.0 \mathrm{mag}$ star in the slit mask with only two 1200 s exposures (stars with numbers beginning with C30), there are roughly 6000 detected electrons per spectral pixel $(1.0 \AA)$ in the region of the $\mathrm{G}$ band of $\mathrm{CH}$ and roughly $500 \mathrm{de}-$ tected electrons per spectral pixel in the blue continuum bandpass for the NH feature. Thus, the uncertainties in the measured $\mathrm{CH}$ and $\mathrm{NH}$ indices are not dominated by Poisson statistics, even for the faintest stars in our sample in M15, but rather by the various instrumental issues described above.

In addition to the primary sample described above, since these fields are rather crowded, other stars sometimes serendipitously fell into the slitlets. If they were bright enough, their spectra were also reduced. We refer to the latter as the secondary sample. As might be expected from the luminosity function, most of the secondary sample consists of stars at or just below the main-sequence turnoff.

\subsection{Membership}

The Galactic latitude of M15 is only $-27^{\circ} .3$. Even though our fields are as close to the center of the cluster as possible, the cluster is more distant than ones we previously studied, and our sample consists of faint stars. Establishing membership is a concern. The primary basis for determining membership is through the spectra. M15 is a very metal-poor cluster, so these moderateresolution spectra are in themselves capable of confirming membership. Table 1 gives the $V, I, J, K$ photometry for the 68 sample stars we believe to be members of M15. Three nonmembers were culled from our sample, as their spectra show absorption features much stronger than expected; they are listed at the end of the table.

The CMDs can also be used to eliminate nonmembers. $B, V$ colors were used to select the primary sample, but they (and other colors) can provide constraints for the secondary stars. Figure 1 shows the $V, I$ and $V, K$ CMDs for our sample in M15 with a $12 \mathrm{Gyr}$ isochrone with $[\mathrm{Fe} / \mathrm{H}]-2.3$ dex from Yi et al. (2001) superposed in each case. The star at the lower left is a hot horizontal-branch star; it is part of the secondary sample. The spectroscopic nonmembers are indicated by large open circles; they all lie on the cluster isochrone in the $V-K \mathrm{CMD}$, and two do for the $V-I \mathrm{CMD}$, with the third only slightly off it. Thus, photometry alone, while it can eliminate many nonmembers of M15 from our sample selected for spectroscopy, is not sufficient in itself.

The magnitude of the radial velocity of M15 is sufficiently high to establish membership through measurements on the red spectra, which are of higher spectral resolution than those from LRIS-B with the adopted instrument configuration. Given the extreme metal deficiency of the stars and the foreground reddening, which produces easily detectable interstellar $\mathrm{Na} \mathrm{D}$ lines, we rely exclusively on $\mathrm{H} \alpha$ for this purpose. Thus, these $v_{r}$ measurements are not of high accuracy, with typical uncertainty of $\pm 30 \mathrm{~km} \mathrm{~s}^{-1}$. A histogram of the radial velocities for 50 stars from our sample is shown in Figure 2. The three spectroscopic nonmembers culled from our sample are indicated. This figure demonstrates that the vast majority of the stars in our sample are members of M15.

The spectra of star C29445_0952 ( $V=16.88)$ in our sample are more extended along the slit than that of a point source and clearly indicate that there are two different stars contributing. The photometric database and the LRIS alignment images were checked; this object turns out to be a close pair of separation 0.8 with a brightness difference of 2 mag. It was not possible to separate the contributions of each to the spectra, so data for this object are included in the tables but not shown in any of the figures.

Figure 3 shows the region of the $3360 \AA$ band of NH in the spectra of two of the stars in the primary sample in M15. These stars have essentially the same stellar parameters $\left(T_{\text {eff }} \sim 5200 \mathrm{~K}\right.$ and $\log g \sim 2.8$ dex) lying at about the same place in the cluster $\mathrm{CMD}$, yet their NH bands differ strongly. From this figure alone we can anticipate one of the key results of our work: the large 
TABLE 1

Photometry for the M15 Sample

\begin{tabular}{|c|c|c|c|c|}
\hline $\mathrm{ID}^{\mathrm{a}}$ & $\begin{array}{c}V \\
\text { (mag) }\end{array}$ & $\begin{array}{c}I \\
(\mathrm{mag})\end{array}$ & $\begin{array}{c}J \\
(\mathrm{mag})\end{array}$ & $\begin{array}{c}K \\
(\mathrm{mag})^{\mathrm{b}}\end{array}$ \\
\hline C29363_0823 ............... & 18.03 & 17.17 & 16.61 & 16.05 \\
\hline C29387_0716.............. & 18.27 & 17.40 & $\ldots$ & $\ldots$ \\
\hline C29388_0634.............. & 16.74 & 15.77 & 15.16 & 14.58 \\
\hline C29396_0843 ............... & 17.51 & 16.61 & 16.01 & 15.49 \\
\hline C29401_0908 ............... & 17.70 & 16.81 & 16.21 & 15.68 \\
\hline C29408_1003 ............... & 18.00 & 17.15 & 16.52 & 16.07 \\
\hline C29413_1023 ................ & 17.32 & 16.46 & 15.81 & 15.34 \\
\hline C29417_0953 ............... & 18.32 & 17.50 & 16.08 & 16.41 \\
\hline C29413_1021 ............... & 18.92 & 18.33 & 14.10 & 13.55 \\
\hline C29424_0729 ${ }^{\mathrm{c}} \ldots \ldots \ldots \ldots$ & 17.83 & 17.97 & $\ldots$ & $\ldots$ \\
\hline C29426_0727 .............. & 16.99 & 16.03 & 15.46 & 14.92 \\
\hline C29429_0801 .............. & 16.86 & 15.94 & 15.37 & 14.84 \\
\hline C29442_0710 ............... & 17.32 & 16.41 & 15.86 & 15.37 \\
\hline C29443_0958.............. & 20.83 & 20.00 & $\ldots$ & $\ldots$ \\
\hline C29444_0618.............. & 18.60 & 17.85 & 17.34 & 16.91 \\
\hline C29445_0952 ${ }^{\mathrm{d}} \ldots \ldots \ldots \ldots$ & 16.88 & 15.90 & 15.16 & 14.63 \\
\hline C29448_0655 .............. & 19.51 & $\ldots$ & $\ldots$ & $\ldots$ \\
\hline C29448_0824_............. & 16.52 & 15.53 & 14.88 & 14.33 \\
\hline C29455_0524............... & 18.60 & 17.78 & 17.28 & $\ldots$ \\
\hline C29457_0518 .............. & 19.27 & 18.68 & $\ldots$ & $\ldots$ \\
\hline C29461_0905 ................ & 19.13 & 18.60 & $\ldots$ & 18.13 \\
\hline C29464_0644_............... & 16.93 & 15.98 & 15.42 & 14.90 \\
\hline C29465_0859............. & 16.91 & 15.89 & 15.26 & 14.72 \\
\hline C29475_0512 ............. & 18.52 & 17.71 & 17.21 & 16.83 \\
\hline C29485_0436.............. & 18.25 & 17.33 & 16.76 & 16.25 \\
\hline C29485_0638 & 17.35 & 16.50 & 15.95 & 15.50 \\
\hline C29491_0504_............. & 17.51 & 16.62 & 15.97 & 15.46 \\
\hline C29504_0630.............. & 18.41 & 17.67 & 17.21 & 16.72 \\
\hline C29523_0432 .............. & 16.98 & 16.03 & 15.35 & 14.80 \\
\hline C29533_0714 .............. & 19.26 & 18.66 & $\ldots$ & $\ldots$ \\
\hline C29534_0711 ............... & 17.72 & 16.84 & 16.27 & 15.77 \\
\hline C29549_0416.............. & 17.86 & 16.97 & 16.27 & 15.76 \\
\hline C29560_0528 .............. & 18.42 & 17.60 & 16.97 & 16.46 \\
\hline C29573_0454.............. & 17.60 & 16.66 & 16.00 & 15.51 \\
\hline C29582_0433 ............... & 16.61 & 15.65 & 14.89 & 14.34 \\
\hline C30029_1248.............. & 17.07 & 16.17 & 15.46 & 14.94 \\
\hline C30048_1355 .............. & 18.17 & 17.24 & 16.63 & 16.17 \\
\hline C30051_1331 ................ & 18.26 & 17.39 & 16.72 & 16.24 \\
\hline C30054_1253............... & 16.67 & 15.73 & 15.02 & 14.49 \\
\hline C30056_1227 ............... & 18.40 & 17.59 & 17.06 & 16.63 \\
\hline C30069_1254.............. & 17.55 & 16.64 & 15.91 & 15.39 \\
\hline C30076_1230.............. & 17.46 & 16.56 & 15.88 & 15.35 \\
\hline C30093_1130 .............. & 18.09 & 17.25 & 16.58 & 16.15 \\
\hline C30095_1227_............. & 18.21 & 17.51 & $\ldots$ & 16.16 \\
\hline C30096_1213 .............. & 16.92 & 16.00 & 15.26 & 14.70 \\
\hline C30102_1201 ............... & 17.75 & 16.90 & 16.21 & $15.74(0.05)$ \\
\hline C30108_0858 ................ & 16.92 & 15.97 & 15.39 & 14.81 \\
\hline C30108_1109 .............. & 18.47 & 17.73 & 17.24 & 16.91 \\
\hline C30114_1055 .............. & 18.18 & 17.34 & 16.86 & 16.35 \\
\hline C30116_1015 .............. & 17.53 & 16.63 & 16.05 & 15.51 \\
\hline C30116_1219 .............. & 16.69 & 15.79 & 15.09 & 14.55 \\
\hline C30123_1138 .............. & 18.27 & 17.41 & 16.89 & $16.38(0.06)$ \\
\hline C30124_0922 ............... & 18.39 & 17.67 & 17.26 & 16.84 \\
\hline C30128_0840 & 17.85 & $\ldots$ & 17.63 & $17.65(0.30)$ \\
\hline C30129_0946.............. & 18.05 & 17.22 & 16.61 & 16.08 \\
\hline C30130_0945 ............... & 19.53 & 19.00 & $\ldots$ & $\ldots$ \\
\hline C30130_1008 ............... & 18.10 & 17.25 & 16.61 & 16.16 \\
\hline C30131_0800.............. & 17.02 & 16.11 & 15.44 & 14.86 \\
\hline C30131_0829.............. & 16.70 & 15.79 & 15.10 & 14.50 \\
\hline C30141_0819............... & 18.17 & 17.33 & 16.72 & 16.18 \\
\hline C30146_0829.............. & 18.28 & 17.47 & 16.85 & 16.35 \\
\hline C30148_0825 .............. & 15.37 & 14.37 & 13.62 & 12.97 \\
\hline C30149_1010.............. & 18.74 & $\ldots$ & 17.53 & 16.94 \\
\hline C30159_1044_............ & 16.76 & 15.81 & 15.18 & 14.61 \\
\hline
\end{tabular}

TABLE 1 - Continued

\begin{tabular}{|c|c|c|c|c|}
\hline $\mathrm{ID}^{\mathrm{a}}$ & $\begin{array}{c}V \\
\text { (mag) }\end{array}$ & $\begin{array}{c}I \\
(\mathrm{mag})\end{array}$ & $\begin{array}{c}J \\
\text { (mag) }\end{array}$ & $\begin{array}{c}K \\
(\mathrm{mag})^{\mathrm{b}}\end{array}$ \\
\hline C30165_0939................ & 18.47 & 17.67 & 17.09 & 16.56 \\
\hline C30179_0938_................. & 18.18 & 17.35 & 16.72 & 16.23 \\
\hline C30199_0745 ................ & 18.26 & 17.47 & 16.82 & 16.30 \\
\hline C30215_0807................. & 17.25 & 16.39 & 15.68 & 15.09 \\
\hline C30247_0911 ................ & 16.66 & $\ldots$ & 15.02 & 14.44 \\
\hline C30251_0915 .................. & 16.72 & $\cdots$ & 15.08 & 14.50 \\
\hline \multicolumn{5}{|c|}{ Nonmembers ${ }^{\mathrm{f}}$} \\
\hline C29393_0829................ & 18.46 & 17.65 & 17.13 & 16.77 \\
\hline C29419_0508................ & 17.88 & 17.00 & 16.45 & 15.93 \\
\hline C30162_0738 ................ & 16.64 & 15.87 & $\ldots$ & $\ldots$ \\
\hline
\end{tabular}

Note.-Table 1 is also available in machine-readable form in the electronic edition of the Astronomical Journal.

a The star names are derived from their J2000.0 coordinates. Star C12345_5432 has coordinates R.A. $=21^{\mathrm{h}} 12^{\mathrm{m}} 34.5$, decl. $=+12^{\circ} 54^{\prime} 32^{\prime \prime}$.

${ }^{\mathrm{b}} \sigma(K)$ from multiple measurements indicated if it exceeds $0.05 \mathrm{mag}$.

c This star is on the extended BHB.

d This object is actually a close pair.

e Crowded.

f Spectrum appears too strongly lined for M15 membership.

scatter in $\mathrm{C}$ and $\mathrm{N}$ abundance we will find among M15 members at the base of the RGB at $17 \mathrm{mag}<V<18.5 \mathrm{mag}$.

\section{MEASUREMENT OF CH AND CN INDICES}

For each spectrum, indices sensitive to absorption by the $4300 \AA \mathrm{CH}$ band were measured as described in Briley \& Cohen (2001). While for the NH band we could have used the doublesided index described in Briley \& Smith (1993) and used by us for our small sample of spectra of M13 subgiants described in Briley et al. (2004a), we were concerned with the decline of the apparent continuum level toward bluer wavelengths in the UV for these Keck LRIS-B spectra. This is presumably due to the wavelength dependence of both the stellar flux and the instrumental efficiency. It is extremely difficult to flux spectra taken through slit masks because of the varying slit losses and the possibility of atmospheric dispersion affecting the spectra, although the latter was, as discussed earlier, not a concern here. Carrying out the observations with the length of the slit set to the parallactic angle, which is the usual method for eliminating atmospheric dispersion for single-slit observations, cannot be used for multislit observations, as the position angle is fixed by the design of the slit mask. It is for these reasons that no attempt was made to flux the spectra.

Since the stars are so metal-poor, there are relatively few detected features in the region between 3300 and $3430 \AA$ besides the desired NH band. Thus, we decided to normalize the stellar continuum in the spectrum of each star, then find the absorption within the NH feature bandpass. The continuum-fitting approach adopted here allows for a direct comparison between indices measured from the spectra and those computed from theoretical spectral synthesis without the need for any slope corrections. The feature bandpass for $\mathrm{NH}$ adopted here was 3354$3375 \AA$ (in the rest frame of M15). This was done by fitting a second-order polynomial to the bandpass $3300-3430 \AA$, masking out the region of the $\mathrm{NH}$ band. The polynomial fitting used a $6 \sigma$ high and $3 \sigma$ low clipping, running over a 5 pixel average. There was little change in the measured NH indices as compared to simply applying the two-sided continuum and feature bandpasses. This reflects our educated guess for the choice of the 


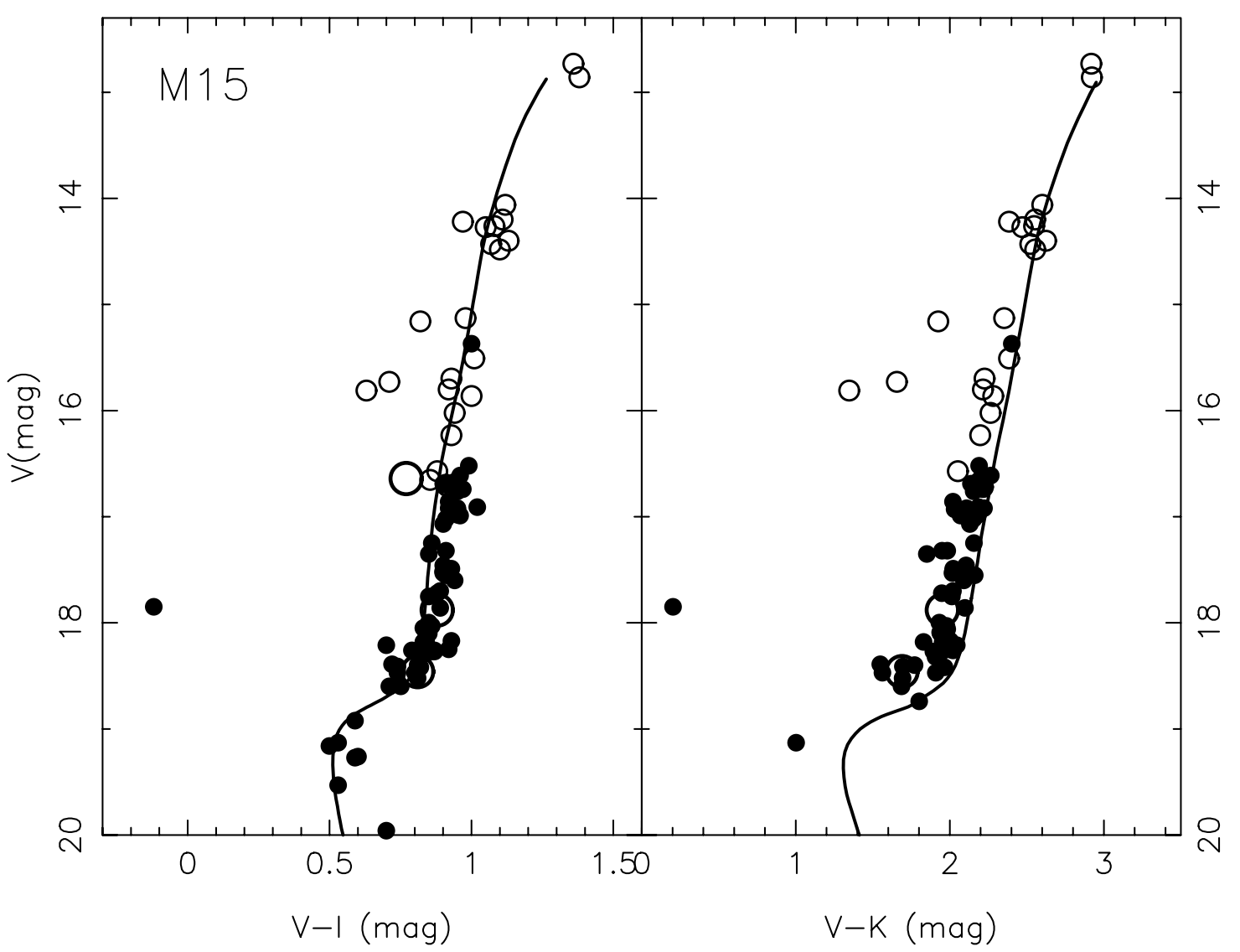

Fig. 1.- $V, I$ and $V, K$ CMDs for M15. The sample stars are indicated by filled circles. The large open circles denote spectroscopic nonmembers. Small open circles denote the members of a Keck HIRES sample to be discussed in J. B. Cohen \& J. Meléndez (2005, in preparation). A $12 \mathrm{Gyr}$, [Fe/H] -2.3 dex isochrone from Yi et al. (2001) is also shown shifted to our adopted cluster distance and reddening.

continuum bandpasses, which are more or less symmetrically distributed about the center of the feature bandpass.

The measured indices are listed in Table 2 and plotted in Figures 4 and 5 . The upper axis of these figures is $M_{V}^{0}$, and the RGB bump in M15 occurs at $V=15.41 \pm 0.04$ mag (Zoccali et al. 1999). Approaching the main-sequence turnoff, $T_{\text {eff }}$ suddenly increases, and the molecular bands we study here become

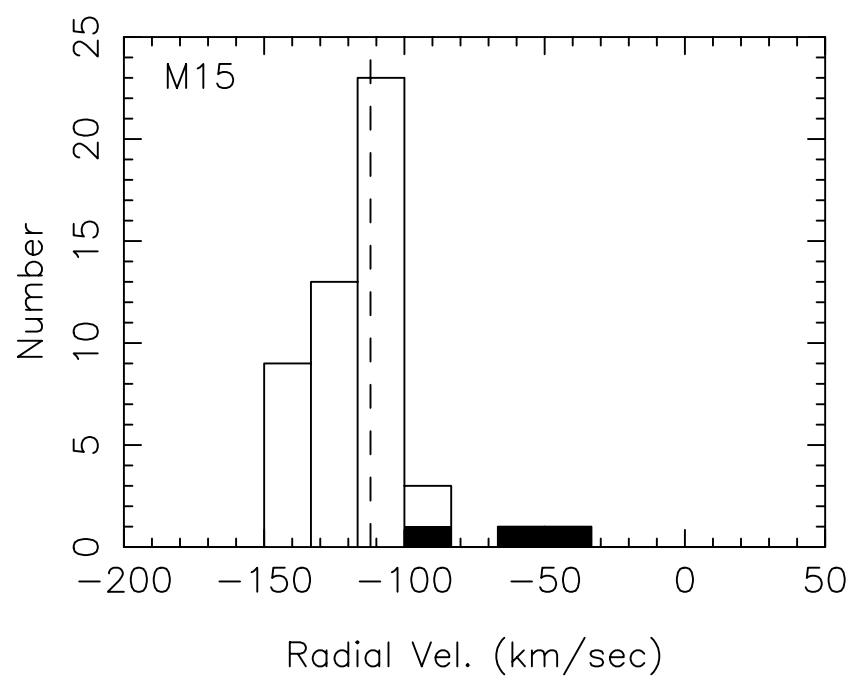

Fig. 2.-Histogram of radial velocities for 50 stars in our sample in M15. The filled area denotes the three nonmember stars based on their spectra. The dashed vertical line indicates the systemic velocity of the cluster.

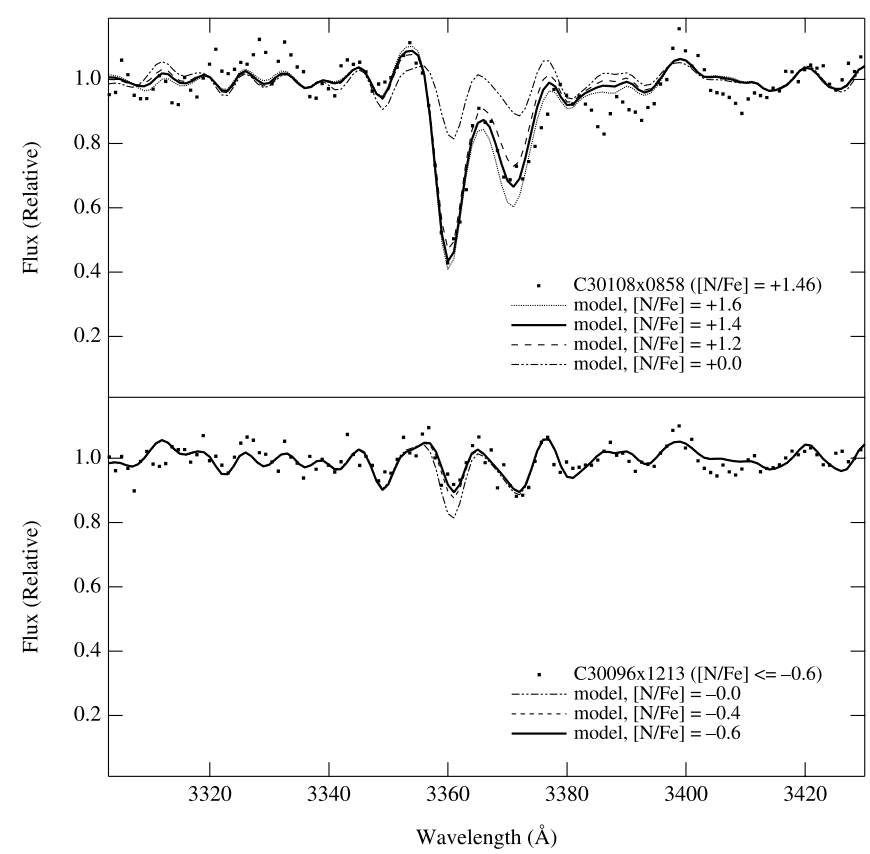

FIG. 3.-LRIS-B spectra of two members of M15 in the region of the NH band (squares). The stars are essentially identical in $V$ magnitude $(V=16.92)$ and $V-I$ colors and are both located at the base of the RGB. Synthetic spectra with various values of $[\mathrm{N} / \mathrm{Fe}]$ are superposed. 
TABLE 2

Deduced C and N Abundances for M15 Sample

\begin{tabular}{|c|c|c|c|c|c|c|c|c|c|}
\hline Star & $\begin{array}{c}V \\
(\mathrm{mag})\end{array}$ & $\begin{array}{l}B-V \\
(\mathrm{mag})\end{array}$ & $\begin{array}{c}M_{V} \\
(\mathrm{mag})\end{array}$ & $\begin{array}{l}T_{\text {eff }} \\
(\mathrm{K})\end{array}$ & $\begin{array}{l}\log g \\
(\operatorname{dex})\end{array}$ & $\begin{array}{l}\mathrm{CH} \\
(\%)\end{array}$ & $\begin{array}{l}\mathrm{NH} \\
(\%)\end{array}$ & $\begin{array}{c}{[\mathrm{C} / \mathrm{Fe}]} \\
(\mathrm{dex})\end{array}$ & $\begin{array}{c}{[\mathrm{N} / \mathrm{Fe}]} \\
(\mathrm{dex})\end{array}$ \\
\hline C30148_0825 ................ & 15.37 & 0.81 & 0.06 & 4939 & 2.05 & $0.123 \pm 0.008$ & $0.244 \pm 0.024$ & $-0.27 \pm 0.06$ & $1.12_{-0.14}^{+0.15}$ \\
\hline C29448_0824.............. & 16.52 & 0.74 & 1.21 & 5143 & 2.60 & $0.106 \pm 0.007$ & $0.013 \pm 0.010$ & $-0.11 \pm 0.06$ & $\leq-0.6$ \\
\hline C29582_0433 .............. & 16.61 & 0.74 & 1.30 & 5157 & 2.65 & $0.099 \pm 0.008$ & $0.187 \pm 0.016$ & $-0.15_{-0.07}^{+0.06}$ & $1.0 \overline{2} \pm 0.09$ \\
\hline C30247_0911 ................ & 16.66 & 0.69 & 1.35 & 5166 & 2.67 & $0.095 \pm 0.006$ & $-0.031 \pm 0.014$ & $-0.17 \pm 0.06$ & $\leq-0.6$ \\
\hline C30054_1253 ........................ & 16.67 & 0.67 & 1.36 & 5167 & 2.67 & $0.102 \pm 0.006$ & $0.190 \pm 0.018$ & $-0.11_{-0.06}^{+0.05}$ & $1.05 \pm 0.10$ \\
\hline 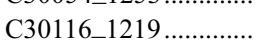 & 16.69 & 0.74 & 1.38 & 5170 & 2.68 & $0.101 \pm 0.006$ & $0.037 \pm 0.017$ & $\begin{array}{l}-0.06 \\
-0.12_{-0.06}^{+0.05}\end{array}$ & $-0.31_{-066}^{+0.30}$ \\
\hline C30131_0829....................... & 16.70 & 0.73 & 1.39 & 5172 & 2.68 & $0.103 \pm 0.007$ & $0.041 \pm 0.015$ & $-0.10_{-0.06}^{+0.06}$ & $-0.23_{-0.43}^{+0.66}$ \\
\hline C29388_0634 ....................... & 16.74 & 0.70 & 1.43 & 5178 & 2.71 & $0.104 \pm 0.004$ & $0.104 \pm 0.012$ & $-0.08 \pm 0.03$ & $0.50_{-0.10}^{+0.43}$ \\
\hline C30159_1044............... & 16.76 & 0.74 & 1.45 & 5181 & 2.72 & $0.113 \pm 0.008$ & $0.115 \pm 0.028$ & $-0.01_{-0.06}^{+0.05}$ & $0.59_{-0.22}^{+0.10}$ \\
\hline C29429_0801 .............. & 16.86 & 0.69 & 1.55 & 5195 & 2.77 & $0.082 \pm 0.005$ & $0.028 \pm 0.016$ & $-0.27 \pm 0.06$ & $-0.54_{-0.82}^{+0.40}$ \\
\hline C29445_0952 ${ }^{\mathrm{a}} \ldots \ldots \ldots \ldots$ & 16.88 & 0.75 & 1.57 & 5198 & 2.78 & $0.059 \pm 0.004$ & $0.071 \pm 0.024$ & & \\
\hline C29465_0859............. & 16.91 & 0.68 & 1.60 & 5203 & 2.79 & $0.100 \pm 0.006$ & $0.247 \pm 0.015$ & $-0.09 \pm 0.05$ & $1.39_{-0.08}^{+0.09}$ \\
\hline C30108_0858_............... & 16.92 & 0.71 & 1.61 & 5205 & 2.79 & $0.061 \pm 0.004$ & $0.259 \pm 0.017$ & $-0.58_{-0.10}^{+0.08}$ & $1.46 \pm 0.10$ \\
\hline C30096_1213 ............... & 16.92 & 0.63 & 1.61 & 5205 & 2.79 & $0.124 \pm 0.009$ & $0.025 \pm 0.019$ & $0.09 \pm 0.06$ & $\leq-0.6$ \\
\hline C29464_0644 ............... & 16.93 & 0.71 & 1.62 & 5207 & 2.80 & $0.077 \pm 0.006$ & $0.213 \pm 0.016$ & $-0.32_{-0.09}^{+0.07}$ & $1.21 \pm 0.09$ \\
\hline C29523_0432 .............. & 16.98 & 0.69 & 1.67 & 5215 & 2.82 & $0.116 \pm 0.009$ & $0.066 \pm 0.018$ & $0.05_{-0.07}^{+0.06}$ & $0.19_{-023}^{+0.19}$ \\
\hline 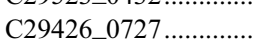 & 16.99 & 0.69 & 1.68 & 5216 & 2.82 & $0.081 \pm 0.006$ & $0.173 \pm 0.014$ & $-0.25_{-0.07}^{+0.07}$ & $0.99 \pm 0.08$ \\
\hline 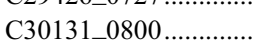 & 17.02 & 0.72 & 1.71 & 5220 & 2.84 & $0.087 \pm 0.003$ & $\ldots$ & $\begin{array}{l}-0.18_{-0.05}^{+0.07} \\
-0.04\end{array}$ & 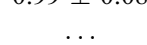 \\
\hline 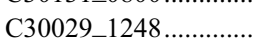 & 17.07 & 0.68 & 1.76 & 5227 & 2.86 & $0.089 \pm 0.004$ & $0.197 \pm 0.021$ & $-0.15_{-0.05}^{+0.05}$ & $1.14 \pm 0.12$ \\
\hline C30215_0807 ................... & 17.25 & 0.65 & 1.94 & 5256 & 2.95 & $0.117 \pm 0.008$ & $0.081 \pm 0.017$ & $0.11_{-0.06}^{+0.05}$ & $0.38_{-0.17}^{+0.15}$ \\
\hline C29442_0710........................ & 17.32 & 0.68 & 2.01 & 5266 & 2.97 & $0.056 \pm 0.004$ & $0.228 \pm 0.015$ & $-0.61_{-0.10}^{+0.09}$ & $1.34 \pm 0.08$ \\
\hline C29413_1023 ............... & 17.32 & 0.69 & 2.01 & 5266 & 2.97 & $0.154 \pm 0.010$ & $0.317 \pm 0.016$ & $0.33 \pm 0.06$ & $1.94_{-0.15}^{*}$ \\
\hline C29485_0638.............. & 17.35 & 0.61 & 2.04 & 5270 & 2.99 & $0.065 \pm 0.004$ & $0.113 \pm 0.018$ & $-0.42_{-0.08}^{+0.07}$ & $0.65_{-0.14}^{+0.13}$ \\
\hline C30076_1230_............... & 17.46 & 0.60 & 2.15 & 5288 & 3.04 & $0.083 \pm 0.006$ & $0.257 \pm 0.025$ & $-0.15_{-0.08}^{+0.08}$ & $1.52_{-0.14}^{+0.14}$ \\
\hline C29491_0504 .............. & 17.51 & 0.64 & 2.20 & 5295 & 3.06 & $0.098 \pm 0.008$ & $0.078 \pm 0.022$ & $0.00 \pm 0.07$ & $0.39_{-0.24}^{+0.14}$ \\
\hline C29396_0843 ............... & 17.51 & 0.71 & 2.20 & 5295 & 3.06 & $0.089 \pm 0.002$ & $0.008 \pm 0.019$ & $-0.080 \pm 0.02$ & $\leq-0.60$ \\
\hline C30116_1015 ................. & 17.53 & 0.71 & 2.22 & 5298 & 3.07 & $0.080 \pm 0.006$ & $0.011 \pm 0.027$ & $-0.18_{-0.08}^{+0.07}$ & $\leq-0.60$ \\
\hline C30069_1254 ........................ & 17.55 & 0.61 & 2.24 & 5301 & 3.08 & $0.067 \pm 0.005$ & $0.116 \pm 0.021$ & $-0.36 \pm 0.08$ & $0.70_{-0.16}^{+0.14}$ \\
\hline C29573_0454_.............. & 17.60 & 0.68 & 2.29 & 5310 & 3.10 & $0.115 \pm 0.009$ & $0.349 \pm 0.025$ & $0.14 \pm 0.06$ & $\geq+2.00$ \\
\hline C29401_0908_............... & 17.70 & 0.68 & 2.39 & 5327 & 3.15 & $0.062 \pm 0.005$ & $0.220 \pm 0.019$ & $-0.38 \pm 0.07$ & $1 . \overline{35} \pm 0.11$ \\
\hline C29534_0711 .............. & 17.72 & 0.66 & 2.41 & 5330 & 3.16 & $0.070 \pm 0.005$ & $-0.046 \pm 0.029$ & $-0.27_{-0.08}^{+0.07}$ & $\leq-0.60$ \\
\hline C30102_1201 ................ & 17.75 & 0.62 & 2.44 & 5336 & 3.17 & $0.046 \pm 0.004$ & $0.243 \pm 0.044$ & $-0.81_{-0.20}^{+0.08}$ & $1.49_{-0.24}^{+0.26}$ \\
\hline C29424_0729 & 17.85 & -0.08 & 2.54 & $>10000$ & $\ldots$ & $0.004 \pm 0.002$ & $\ldots$ & $\ldots$ & $\ldots$ \\
\hline C29549_0416............... & 17.86 & 0.64 & 2.55 & 5356 & 3.23 & $0.079 \pm 0.006$ & $-0.002 \pm 0.032$ & $-0.12 \pm 0.07$ & $\leq-0.6$ \\
\hline C29408_1003 .............. & 18.00 & 0.67 & 2.69 & 5388 & 3.30 & $0.089 \pm 0.006$ & $0.031 \pm 0.018$ & $0.02_{-0.06}^{+0.05}$ & $-0.17_{*}^{+0.32}$ \\
\hline C29363_0823 ................ & 18.03 & 0.67 & 2.72 & 5396 & 3.31 & $0.075 \pm 0.006$ & $0.031 \pm 0.024$ & $-0.13_{-0.07}^{+0.06}$ & $-0.16_{*}^{* 0.40}$ \\
\hline C30129_0946............. & 18.05 & 0.65 & 2.74 & 5401 & 3.32 & $0.058 \pm 0.005$ & $0.297 \pm 0.046$ & $-0.36_{-0.11}^{+0.09}$ & $1.88_{-0.30}^{*}$ \\
\hline C30093_1130 ...................... & 18.09 & 0.58 & 2.78 & 5413 & 3.34 & $0.069 \pm 0.005$ & $0.023 \pm 0.031$ & $-0.17_{-0.08}^{+0.06}$ & $-0.38_{*}^{+0.64}$ \\
\hline C30130_1008_................ & 18.10 & 0.68 & 2.79 & 5416 & 3.34 & $0.049 \pm 0.004$ & $0.146 \pm 0.035$ & $-0.56_{-0.13}^{+0.11}$ & $1.01_{-0.23}^{* 0.21}$ \\
\hline 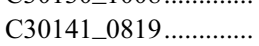 & 18.17 & 0.65 & 2.86 & 5440 & 3.38 & $0.067 \pm 0.007$ & $0.041 \pm 0.049$ & $-0.27_{-0.13}^{+0.13}$ & $0.09_{*}^{+0.23}$ \\
\hline C30048_1355 ............................. & 18.17 & 0.70 & 2.86 & 5440 & 3.38 & $0.061 \pm 0.006$ & $0.045 \pm 0.041$ & $-0.17_{-0.11}^{+0.09}$ & $0.16_{*}^{+0.46}$ \\
\hline 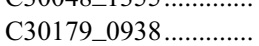 & 18.18 & 0.64 & 2.87 & 5444 & 3.38 & $0.044 \pm 0.004$ & $0.173 \pm 0.028$ & $-0.69_{-017}^{+0.13}$ & $1.19 \pm 0.16$ \\
\hline C30114_1055 ........................ & 18.18 & 0.62 & 2.87 & 5444 & 3.38 & $0.056 \pm 0.005$ & $0.151 \pm 0.028$ & $-0.36_{-0.09}^{+0.17}$ & $1.07_{-0.18}^{+0.16}$ \\
\hline C30095_1227 ................. & 18.21 & 0.57 & 2.90 & 5457 & 3.41 & $0.074 \pm 0.006$ & $-0.018 \pm 0.034$ & $-0.06_{-0.07}^{+0.106}$ & $\leq-0.6$ \\
\hline C29485_0436............... & 18.25 & 0.65 & 2.94 & 5475 & 3.43 & $0.081 \pm 0.007$ & $0.007 \pm 0.032$ & $0.03 \pm 0.07$ & $\leq-0.6$ \\
\hline C30199_0745 ............... & 18.26 & 0.60 & 2.95 & 5480 & 3.44 & $0.052 \pm 0.004$ & $-0.002 \pm 0.047$ & $-0.41_{-0.11}^{+0.09}$ & $\overline{\leq}-0.6$ \\
\hline C30051_1331_............ & 18.26 & 0.70 & 2.95 & 5480 & 3.44 & $0.085 \pm 0.006$ & $0.019 \pm 0.035$ & $0.08 \pm 0.06$ & $-0.43_{*}^{+0.77}$ \\
\hline C30123_1138_............... & 18.27 & 0.57 & 2.96 & 5485 & 3.44 & $0.109 \pm 0.008$ & $0.254 \pm 0.034$ & $0.29_{-0.06}^{+0.05}$ & $1.69_{-0.19}^{+0.22}$ \\
\hline C29387_0716............... & 18.27 & 0.64 & 2.96 & 5485 & 3.44 & $0.043 \pm 0.003$ & $0.234 \pm 0.022$ & $-0.67 \pm 0.14$ & $1.58 \pm 0.12$ \\
\hline C30146_0829.............. & 18.28 & 0.64 & 2.97 & 5491 & 3.45 & $0.058 \pm 0.004$ & $0.088 \pm 0.028$ & $-0.26 \pm 0.08$ & $0.68_{-0.26}^{+0.21}$ \\
\hline 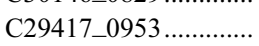 & 18.32 & 0.63 & 3.01 & 5515 & 3.47 & $0.061 \pm 0.004$ & $0.109 \pm 0.019$ & $-0.17_{-0.08}^{+0.07}$ & $0.87_{-0.14}^{+0.13}$ \\
\hline C30124_0922 ............... & 18.39 & 0.52 & 3.08 & 5574 & 3.52 & $0.024 \pm 0.002$ & $-0.010 \pm 0.032$ & $\ldots$ & \\
\hline 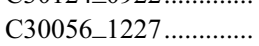 & 18.40 & 0.56 & 3.09 & 5585 & 3.53 & $0.024 \pm 0.005$ & $0.226 \pm 0.040$ & $\begin{array}{l}\cdots \\
\cdots\end{array}$ & $1.64_{-0.22}^{+0.23}$ \\
\hline 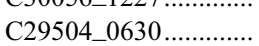 & 18.41 & 0.54 & 3.10 & 5596 & 3.53 & $0.024 \pm 0.003$ & $0.099 \pm 0.024$ & 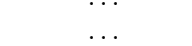 & $0.89_{-020}^{+0.22}$ \\
\hline 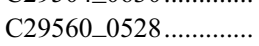 & 18.42 & 0.62 & 3.11 & 5609 & 3.54 & $0.049 \pm 0.004$ & $0.120 \pm 0.042$ & $-0.28_{-0.12}^{+0.09}$ & $1.05_{-0.32}^{+0.20}$ \\
\hline C30165_0939............. & 18.47 & 0.58 & 3.16 & 5690 & 3.59 & $0.020 \pm 0.015$ & $0.345 \pm 0.059$ & $\ldots$ & $\geq+2.0$ \\
\hline C30108_1109 ................ & 18.47 & 0.54 & 3.16 & 5690 & 3.59 & $0.017 \pm 0.003$ & $0.026 \pm 0.031$ & $\ldots$ & $0.11_{*}^{+0.51}$ \\
\hline C29475_0512 ............... & 18.52 & 0.55 & 3.21 & 5802 & 3.65 & $0.026 \pm 0.003$ & $0.131 \pm 0.028$ & $\ldots$ & $1.33_{-0.19}^{+0.17}$ \\
\hline C29455_0524 ............. & 18.60 & 0.56 & 3.29 & 6000 & 3.75 & $0.040 \pm 0.003$ & $0.030 \pm 0.030$ & $0.06_{-0.12}^{+0.08}$ & $0.68_{*}^{+0.39}$ \\
\hline C29444_0618.............. & 18.60 & 0.54 & 3.29 & 6000 & 3.75 & $0.025 \pm 0.003$ & $0.081 \pm 0.028$ & $\ldots$ & $1.26_{-0.26}^{+0.21}$ \\
\hline C29413_1021 .............. & 18.92 & 0.45 & 3.61 & 6477 & 4.02 & $0.028 \pm 0.003$ & $0.000 \pm 0.024$ & $\ldots$ & $0.68_{*}^{+0.78}$ \\
\hline C29461_0905 ..................... & 19.13 & 0.43 & 3.82 & 6569 & 4.12 & $0.027 \pm 0.002$ & & & \\
\hline 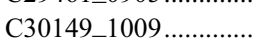 & 19.16 & 0.50 & 3.85 & 6575 & 4.14 & $0.028 \pm 0.005$ & $-0.010 \pm 0.046$ & $\ldots$ & \\
\hline
\end{tabular}


TABLE 2-Continued

\begin{tabular}{|c|c|c|c|c|c|c|c|c|c|}
\hline Star & $\begin{array}{c}V \\
(\mathrm{mag})\end{array}$ & $\begin{array}{l}B-V \\
\text { (mag) }\end{array}$ & $\begin{array}{c}M_{V} \\
\text { (mag) }\end{array}$ & $\begin{array}{l}T_{\text {eff }} \\
(\mathrm{K})\end{array}$ & $\begin{array}{l}\log g \\
(\operatorname{dex})\end{array}$ & $\begin{array}{l}\mathrm{CH} \\
(\%)\end{array}$ & $\begin{array}{l}\mathrm{NH} \\
(\%)\end{array}$ & $\begin{array}{c}{[\mathrm{C} / \mathrm{Fe}]} \\
(\mathrm{dex})\end{array}$ & $\begin{array}{c}{[\mathrm{N} / \mathrm{Fe}]} \\
(\mathrm{dex})\end{array}$ \\
\hline C29533_0714 …......... & 19.26 & $\ldots$ & 3.95 & 6575 & 4.17 & $0.008 \pm 0.005$ & $\ldots$ & & $\ldots$ \\
\hline C29457_0518 .............. & 19.27 & 0.44 & 3.96 & 6581 & 4.18 & $0.022 \pm 0.003$ & $\ldots$ & $-0.46_{*}^{+0.85}$ & $\ldots$ \\
\hline C29448_0655 .............. & 19.51 & 0.44 & 4.20 & 6559 & 4.27 & $0.010 \pm 0.004$ & $\ldots$ & $\ldots$ & $\ldots$ \\
\hline C30130_0945 ............... & 19.53 & 0.45 & 4.22 & 6554 & 4.27 & $0.015 \pm 0.005$ & $-0.005 \pm 0.049$ & $\ldots$ & $\ldots$ \\
\hline C29443_0958................ & 20.83 & 0.60 & 5.52 & 5997 & 4.58 & $0.027 \pm 0.004$ & $-0.011 \pm 0.038$ & $\ldots$ & $\ldots$ \\
\hline
\end{tabular}

Noте.-Table 2 is also available in machine-readable form in the electronic edition of the Astronomical Journal.

a This object is a close pair.

${ }^{\mathrm{b}}$ Hot star on extended horizontal branch in M15.

much weaker; they are effectively undetectable in the present spectra. This produces the sharp drop in measured indices apparent at $V \sim 18.5$ in Figure 4. It is not until $\sim 2.5 \mathrm{mag}$ below the main-sequence turnoff that $T_{\text {eff }}$ as cool as $5600 \mathrm{~K}$ is again reached, at which point molecular bands can again be expected to be detectable in M15 main-sequence stars with similar quality spectra to those presented here for its subgiants.

The error bars given in Table 2 (drawn in Fig. 4 at $2 \sigma$ ) have been calculated strictly from Poisson statistics based on the signal present in the feature and continuum bandpasses. The $\mathrm{CH}$ index, in particular, is very robust; independent measurements by each of the authors using both a double-sided index and continuum fitting as described above always agreed to within $2 \%$ for each star in the sample in M15. Every star in the

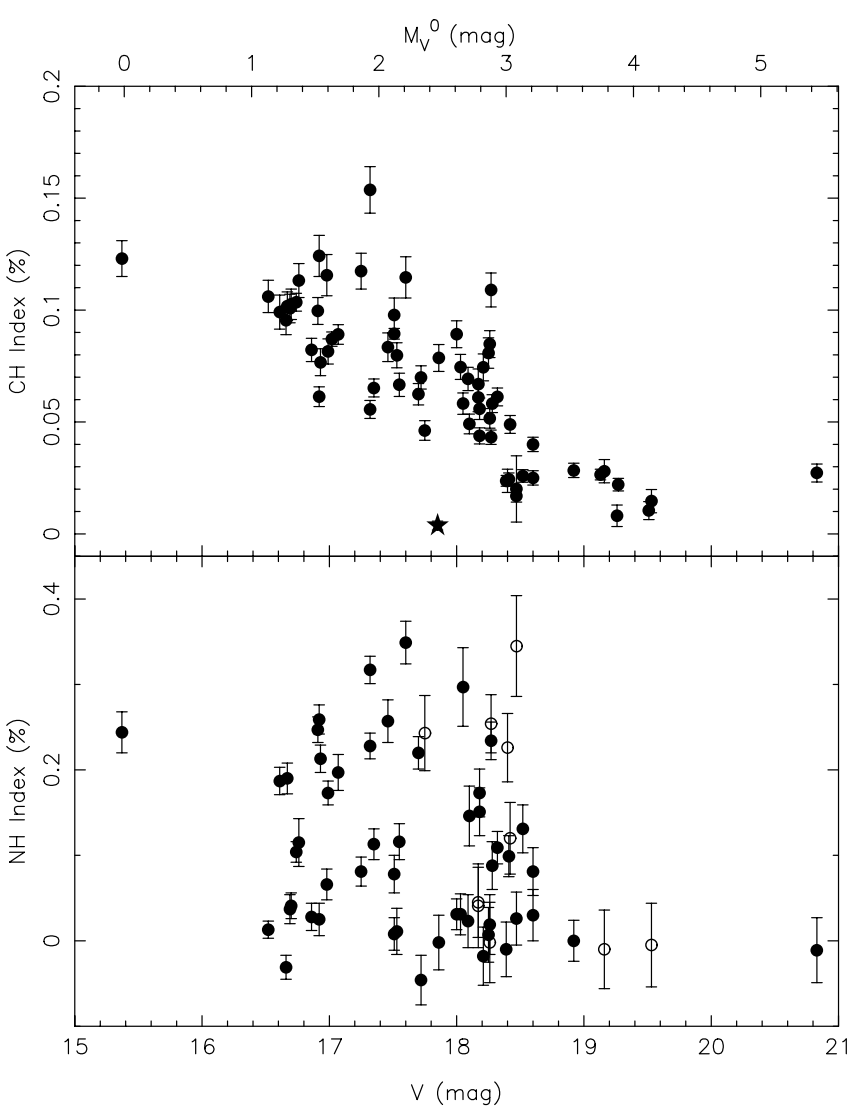

FIG. 4. - Measured $I(\mathrm{CH})$ and $I(\mathrm{NH})$ indices for the program stars as a function of $V$. The top axis is $M_{V}^{0}$. The open circles in $I(\mathrm{NH})$ (bottom) denote the stars with the smallest signal level at $3350 \AA$. Large and significant star-to-star differences exist in both $\mathrm{CH}$ and $\mathrm{NH}$ band strengths among these stars. The decreasing spread in indices with luminosity is the result of increasing temperatures near the mainsequence turnoff. The star denotes the extreme BHB star in our M15 sample. sample has a measurement of $I(\mathrm{CH})$. The value of $I(\mathrm{CH})$ is positive for all the stars in the sample and exceeds $1 \%$ for all stars except C29424_0729 on the extended blue horizontal branch (BHB) and one star near the main-sequence turnoff at $V=$ $19.26 \mathrm{mag}$. We do not expect to see any $\mathrm{CH}$ for the extreme BHB star or the stars just at the turnoff. The measured $I(\mathrm{CH})$ values for them range from $0 \%$ to $4 \%$, with most having $I(\mathrm{CH}) \leq 2 \%$. These include the faintest stars in the sample, and their $I(\mathrm{CH})$ measurements appear valid, again suggesting that the measurements of the $\mathrm{CH}$ indices at all luminosities considered here are robust. As can be seen in Figure 4, substantial star-to-star differences occur among the $I(\mathrm{CH})$ indices for the stars in our M15

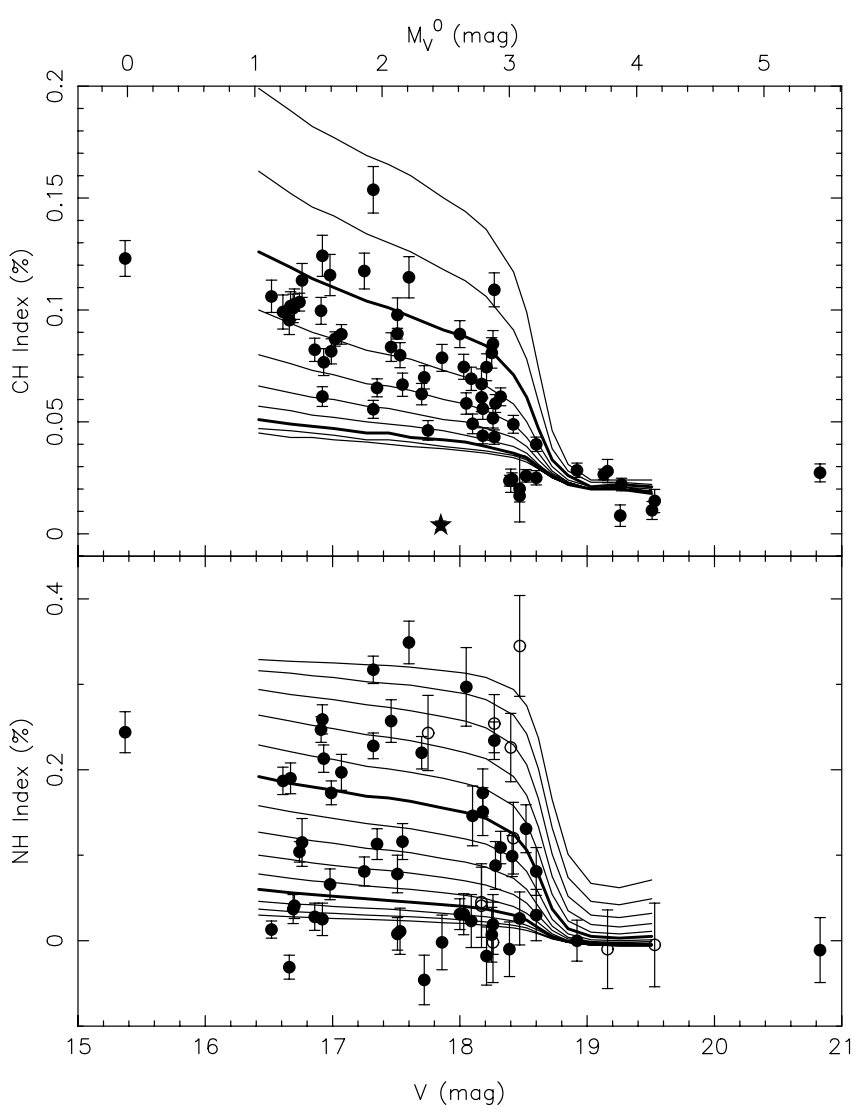

Fig. 5.- - Measured $I(\mathrm{CH})$ and $I(\mathrm{NH})$ indices for the program stars as a function of $V$. The top axis is $M_{V}^{0}$. The model $I(\mathrm{CH})$ and $I(\mathrm{NH})$ are superposed, with thick lines denoting $[\mathrm{C} / \mathrm{Fe}]=0.0$ and -1.0 dex $(t o p)$ and $[\mathrm{N} / \mathrm{Fe}]=0$ and +1.0 dex (bottom). The open circles (bottom) denote the stars with the smallest signal level at $3350 \AA$. Large and significant star-to-star differences exist in both $\mathrm{CH}$ and $\mathrm{NH}$ band strengths among these stars. The decreasing spread in indices with luminosity is the result of increasing temperatures near the main-sequence turnoff. The star denotes the extreme BHB star in our M15 sample. 
TABLE 3

$I(\mathrm{CH})(4305 \AA$ Band) From Synthetic Spectra

\begin{tabular}{|c|c|c|c|c|c|c|c|c|c|c|c|c|}
\hline $\begin{array}{c}T_{\text {eff }} \\
(\mathrm{K})\end{array}$ & $\begin{array}{l}\log g \\
(\operatorname{dex})\end{array}$ & $M_{V}^{0}$ & $\begin{array}{c}{[\mathrm{C} / \mathrm{Fe}]} \\
-1.4 \\
(\mathrm{dex})\end{array}$ & $\begin{array}{c}{[\mathrm{C} / \mathrm{Fe}]} \\
-1.2 \\
(\mathrm{dex})\end{array}$ & $\begin{array}{c}{[\mathrm{C} / \mathrm{Fe}]} \\
-1.0 \\
(\mathrm{dex})\end{array}$ & $\begin{array}{c}{[\mathrm{C} / \mathrm{Fe}]} \\
-0.8 \\
(\mathrm{dex})\end{array}$ & $\begin{array}{c}{[\mathrm{C} / \mathrm{Fe}]} \\
-0.6 \\
(\mathrm{dex})\end{array}$ & $\begin{array}{c}{[\mathrm{C} / \mathrm{Fe}]} \\
-0.4 \\
(\mathrm{dex})\end{array}$ & $\begin{array}{c}{[\mathrm{C} / \mathrm{Fe}]} \\
-0.2 \\
(\mathrm{dex})\end{array}$ & $\begin{array}{c}{[\mathrm{C} / \mathrm{Fe}]} \\
+0.0 \\
(\mathrm{dex})\end{array}$ & $\begin{array}{c}{[\mathrm{C} / \mathrm{Fe}]} \\
+0.2 \\
(\mathrm{dex})\end{array}$ & $\begin{array}{c}{[\mathrm{C} / \mathrm{Fe}]} \\
+0.4 \\
(\mathrm{dex})\end{array}$ \\
\hline $5159 \ldots \ldots \ldots \ldots \ldots \ldots \ldots \ldots \ldots \ldots \ldots$ & 2.57 & 1.031 & 0.045 & 0.047 & 0.051 & 0.057 & 0.066 & 0.080 & 0.100 & 0.126 & 0.162 & 0.199 \\
\hline $5201 \ldots \ldots \ldots \ldots \ldots \ldots \ldots$ & 2.68 & 1.283 & 0.043 & 0.046 & 0.049 & 0.055 & 0.063 & 0.076 & 0.094 & 0.119 & 0.152 & 0.189 \\
\hline $5229 \ldots \ldots \ldots \ldots \ldots \ldots$ & 2.76 & 1.452 & 0.043 & 0.045 & 0.048 & 0.053 & 0.061 & 0.073 & 0.090 & 0.114 & 0.146 & 0.182 \\
\hline $5255 \ldots \ldots \ldots \ldots \ldots \ldots$ & 2.84 & 1.622 & 0.042 & 0.044 & 0.047 & 0.052 & 0.060 & 0.071 & 0.087 & 0.110 & 0.142 & 0.177 \\
\hline $5297 \ldots \ldots \ldots \ldots \ldots \ldots$ & 2.96 & 1.878 & 0.041 & 0.042 & 0.045 & 0.050 & 0.057 & 0.067 & 0.082 & 0.104 & 0.134 & 0.169 \\
\hline $5320 \ldots \ldots$ & 3.04 & 2.052 & 0.040 & 0.042 & 0.045 & 0.049 & 0.056 & 0.066 & 0.080 & 0.101 & 0.130 & 0.165 \\
\hline $5346 \ldots \ldots \ldots \ldots \ldots \ldots \ldots \ldots \ldots \ldots \ldots$ & 3.12 & 2.225 & 0.039 & 0.041 & 0.043 & 0.048 & 0.054 & 0.064 & 0.078 & 0.097 & 0.126 & 0.160 \\
\hline 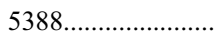 & 3.23 & 2.484 & 0.038 & 0.039 & 0.042 & 0.046 & 0.051 & 0.060 & 0.073 & 0.091 & 0.118 & 0.150 \\
\hline $5418 \ldots \ldots \ldots \ldots \ldots \ldots$ & 3.31 & 2.654 & 0.037 & 0.038 & 0.041 & 0.044 & 0.050 & 0.058 & 0.070 & 0.088 & 0.113 & 0.144 \\
\hline $5454 \ldots \ldots \ldots \ldots \ldots \ldots \ldots \ldots \ldots \ldots \ldots$ & 3.39 & 2.818 & 0.036 & 0.037 & 0.039 & 0.042 & 0.047 & 0.055 & 0.066 & 0.084 & 0.106 & 0.136 \\
\hline $5532 \ldots \ldots \ldots \ldots \ldots \ldots$ & 3.51 & 3.033 & 0.034 & 0.035 & 0.036 & 0.039 & 0.043 & 0.049 & 0.058 & 0.071 & 0.091 & 0.117 \\
\hline $5607 \ldots \ldots \ldots \ldots \ldots$ & 3.57 & 3.136 & 0.032 & 0.033 & 0.034 & 0.036 & 0.039 & 0.043 & 0.050 & 0.061 & 0.078 & 0.099 \\
\hline $5739 \ldots \ldots \ldots \ldots \ldots \ldots \ldots \ldots \ldots$ & 3.66 & 3.229 & 0.029 & 0.029 & 0.030 & 0.031 & 0.033 & 0.036 & 0.040 & 0.047 & 0.059 & 0.074 \\
\hline 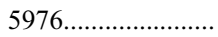 & 3.78 & 3.337 & 0.025 & 0.025 & 0.026 & 0.026 & 0.027 & 0.028 & 0.030 & 0.033 & 0.038 & 0.046 \\
\hline $6217 \ldots \ldots \ldots \ldots \ldots \ldots$ & 3.91 & 3.461 & 0.022 & 0.022 & 0.022 & 0.022 & 0.023 & 0.023 & 0.024 & 0.026 & 0.028 & 0.031 \\
\hline $6387 \ldots \ldots \ldots \ldots \ldots \ldots . . . . . . . . .$. & 4.03 & 3.641 & 0.020 & 0.020 & 0.020 & 0.020 & 0.020 & 0.020 & 0.021 & 0.021 & 0.023 & 0.024 \\
\hline 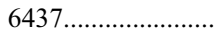 & 4.13 & 3.864 & 0.020 & 0.020 & 0.020 & 0.020 & 0.020 & 0.021 & 0.021 & 0.022 & 0.023 & 0.024 \\
\hline $6412 \ldots \ldots \ldots \ldots \ldots \ldots \ldots \ldots \ldots \ldots \ldots$ & 4.22 & 4.118 & 0.018 & 0.018 & 0.018 & 0.019 & 0.019 & 0.019 & 0.020 & 0.021 & 0.022 & 0.024 \\
\hline
\end{tabular}

sample, even if one considers only stars in similar evolutionary states.

Obtaining high-quality spectra in the region of the $\mathrm{NH}$ band at $3360 \AA$ for such faint stars is not easy. There are five stars with poor-quality spectra in the $\mathrm{NH}$ region for which no measurement of the NH index was attempted. These arose as a result of low signal level, the location of the spectra with respect to the edges of the slit (a problem more common among the secondary stars), or a bright reflection falling on or very near the $\mathrm{NH}$ feature. There are 10 additional stars with low signal level but measured $I(\mathrm{NH})$, which are marked by open circles in Figures 4 and 5; only one of these is brighter than $V=18.1$. Among the stars brighter than $V=18.3$ with measured $I(\mathrm{NH})$, there are five with negative $I(\mathrm{NH})$. Of these, three have $I(\mathrm{NH}) \geq-2 \%$, while the smallest is $-5 \%$. Figure 4 demonstrates that a very substantial range exists in $I(\mathrm{NH})$ among stars of similar evolutionary state for $17<V<18\left(1.6<M_{V}^{0}<2.6\right) \mathrm{mag}$.

In examining Figure 4, aside from the extreme BHB star, there are no obvious anomalous stars in our sample in M15. Furthermore, there is no obvious bimodality among either the $\mathrm{CH}$ or $\mathrm{NH}$ indices. Over a small range in $T_{\text {eff }}$, there appears to be an anticorrelation of the strength of the $\mathrm{CH}$ and $\mathrm{NH}$ bands. The correlation is not perfect, but given the weakness of the molecular features in these low-luminosity stars in M15, it is reasonably convincing. We return to this issue in $\S 6$ once $\mathrm{C}$ and $\mathrm{N}$ abundances are derived for the stars in our M15 sample.

The large range in $\mathrm{C}$ abundances that we suspect to be present in the M15 subgiant sample creates an unusual situation with regard to the expected strength of the $\mathrm{CN}$ features. Normally, since there is more carbon than nitrogen, the $\mathrm{N}$ abundance controls the amount of $\mathrm{CN}$. However, if $\mathrm{C}$ is highly depleted, there can be fewer carbon atoms per unit volume than nitrogen atoms, and $\mathrm{C}$ will control the formation of $\mathrm{CN}$, as suggested by Langer (1985). Since we are using the NH band to deduce N abundances, this is not an issue here.

\section{COMPARISONS WITH SYNTHETIC SPECTRA}

Clearly, the pattern of abundances underlying the $\mathrm{CH}$ and $\mathrm{NH}$ band indices of Figure 4 cannot be interpreted on the basis of band strengths alone; we must turn to models. The technique employed is similar to that of Briley \& Cohen (2001), in which the region of the CMD of interest is fitted by a series of models whose parameters are taken from (in the present case) a $Y^{2}$ (Demarque et al. 2004) $12 \mathrm{Gyr}$ isochrone with $Z=$ $0.000400, Y=0.230800$, and $[\alpha / \mathrm{Fe}]=0.6$. The representative model points are listed in Table 3. Model stellar atmospheres were then generated using the MARCS model atmosphere program (Gustafsson et al. 1975) at the $T_{\text {eff }}, \log g$ of these points. Logarithmic solar abundances of $\mathrm{Fe}, \mathrm{C}, \mathrm{N}$, and $\mathrm{O}$ are assumed to be $7.52,8.62,8.00$, and $8.86 \mathrm{dex}$, respectively, on a scale of $\mathrm{H}=12$. These are somewhat higher than the latest solar abundances inferred from three-dimensional hydrodynamic models by Asplund et al. (2005), but we continue to use them for consistency with our previously published papers in this series. Furthermore, our molecular transition probabilities were scaled to fit the solar spectrum with our adopted solar abundances when they were first adopted (e.g., Bell et al. 1994). From each model, synthetic spectra were calculated using the SSG program (Bell \& Gustafsson 1978, 1989; Gustafsson \& Bell 1979; Bell et al. 1994) and the line list of Tripicco \& Bell (1995). The spectra were calculated from 3200 to $5500 \AA$ in $0.05 \AA$ steps with a microturbulent velocity of $2 \mathrm{~km} \mathrm{~s}^{-1}$, an $[\mathrm{O} / \mathrm{Fe}]$ abundance of $+0.20 \mathrm{dex}$, and ${ }^{12} \mathrm{C} /{ }^{13} \mathrm{C}=10$, but with differing $\mathrm{C}$ and $\mathrm{N}$ abundances. The resulting spectra were then smoothed to the resolution of the observed spectra and the corresponding $I(\mathrm{CH})$ and $I(\mathrm{NH})$ indices measured. By construction, no zero-point shifts were necessary.

The values of $I(\mathrm{CH})$ from 18 sets of models with $[\mathrm{C} / \mathrm{Fe}]$ from -1.4 to +0.4 dex in steps of 0.2 dex are plotted with the observed indices in Figure 5. The value of $I(\mathrm{NH})$ was computed from the same set of models for [N/Fe] from -0.6 to +2.0 dex in steps of 0.2 dex. The corresponding $I(\mathrm{NH})$ values are also shown in the figure. The spread in $[\mathrm{C} / \mathrm{Fe}]$ and $[\mathrm{N} / \mathrm{Fe}]$ among the M15 subgiant branch (SGB) stars appears well represented by this range. Clearly, the star-to-star spread in $\mathrm{N}$ abundances of the stars at the base of the RGB in M15 approaches a factor of 10. The resulting $I(\mathrm{CH})$ and $I(\mathrm{NH})$ indices predicted via synthetic spectra from 
TABLE 4

I(NH) (3360 ̊ B AND) From Synthetic SPeCtra

\begin{tabular}{|c|c|c|c|c|c|c|c|c|c|c|c|c|c|c|}
\hline$M_{V}^{0}$ & $\begin{array}{c}{[\mathrm{N} / \mathrm{Fe}]} \\
-0.6 \\
(\mathrm{dex})\end{array}$ & $\begin{array}{c}{[\mathrm{N} / \mathrm{Fe}]} \\
-0.4 \\
(\mathrm{dex})\end{array}$ & $\begin{array}{c}{[\mathrm{N} / \mathrm{Fe}]} \\
-0.2 \\
(\mathrm{dex})\end{array}$ & $\begin{array}{c}{[\mathrm{N} / \mathrm{Fe}]} \\
+0.0 \\
(\mathrm{dex})\end{array}$ & $\begin{array}{c}{[\mathrm{N} / \mathrm{Fe}]} \\
+0.2 \\
(\mathrm{dex})\end{array}$ & $\begin{array}{c}{[\mathrm{N} / \mathrm{Fe}]} \\
+0.4 \\
(\mathrm{dex})\end{array}$ & $\begin{array}{c}{[\mathrm{N} / \mathrm{Fe}]} \\
+0.8 \\
(\mathrm{dex})\end{array}$ & $\begin{array}{c}{[\mathrm{N} / \mathrm{Fe}]} \\
+1.0 \\
(\mathrm{dex})\end{array}$ & $\begin{array}{c}{[\mathrm{N} / \mathrm{Fe}]} \\
+1.2 \\
(\mathrm{dex})\end{array}$ & $\begin{array}{c}{[\mathrm{N} / \mathrm{Fe}]} \\
+1.4 \\
(\mathrm{dex})\end{array}$ & $\begin{array}{c}{[\mathrm{N} / \mathrm{Fe}]} \\
+1.6 \\
(\mathrm{dex})\end{array}$ & $\begin{array}{c}{[\mathrm{N} / \mathrm{Fe}]} \\
+1.8 \\
(\mathrm{dex})\end{array}$ & $\begin{array}{c}{[\mathrm{N} / \mathrm{Fe}]} \\
+2.0 \\
(\mathrm{dex})\end{array}$ & $\begin{array}{c}{[\mathrm{N} / \mathrm{Fe}]} \\
+2.2 \\
(\mathrm{dex})\end{array}$ \\
\hline $1.031 \ldots \ldots \ldots \ldots$ & 0.030 & 0.037 & 0.046 & 0.060 & 0.078 & 0.100 & 0.127 & 0.158 & 0.192 & 0.229 & 0.264 & 0.294 & 0.316 & 0.329 \\
\hline $1.283 \ldots \ldots \ldots \ldots$ & 0.028 & 0.034 & 0.043 & 0.056 & 0.073 & 0.095 & 0.121 & 0.151 & 0.184 & 0.221 & 0.257 & 0.288 & 0.313 & 0.327 \\
\hline $1.452 \ldots \ldots \ldots \ldots$ & 0.027 & 0.033 & 0.042 & 0.054 & 0.070 & 0.091 & 0.117 & 0.147 & 0.180 & 0.216 & 0.252 & 0.285 & 0.310 & 0.326 \\
\hline $1.622 \ldots$ & 0.026 & 0.032 & 0.040 & 0.052 & 0.068 & 0.088 & 0.114 & 0.143 & 0.176 & 0.212 & 0.248 & 0.282 & 0.308 & 0.325 \\
\hline $1.878 \ldots \ldots \ldots \ldots$ & 0.025 & 0.030 & 0.038 & 0.049 & 0.064 & 0.084 & 0.108 & 0.137 & 0.169 & 0.204 & 0.241 & 0.276 & 0.303 & 0.323 \\
\hline $2.052 \ldots \ldots \ldots \ldots$ & 0.024 & 0.029 & 0.037 & 0.047 & 0.062 & 0.082 & 0.106 & 0.134 & 0.167 & 0.201 & 0.238 & 0.273 & 0.301 & 0.322 \\
\hline $2.225 \ldots \ldots \ldots \ldots$ & 0.023 & 0.028 & 0.035 & 0.045 & 0.060 & 0.079 & 0.103 & 0.131 & 0.163 & 0.197 & 0.234 & 0.269 & 0.299 & 0.320 \\
\hline $2.484 \ldots \ldots \ldots \ldots$ & 0.021 & 0.026 & 0.032 & 0.042 & 0.056 & 0.074 & 0.096 & 0.124 & 0.155 & 0.190 & 0.226 & 0.262 & 0.293 & 0.316 \\
\hline $2.654 \ldots \ldots \ldots \ldots$ & 0.020 & 0.024 & 0.030 & 0.040 & 0.053 & 0.070 & 0.092 & 0.119 & 0.150 & 0.184 & 0.220 & 0.257 & 0.289 & 0.313 \\
\hline $2.818 \ldots \ldots \ldots \ldots$ & 0.018 & 0.022 & 0.028 & 0.037 & 0.049 & 0.065 & 0.086 & 0.113 & 0.143 & 0.177 & 0.213 & 0.249 & 0.282 & 0.308 \\
\hline $3.033 \ldots \ldots \ldots \ldots$ & 0.015 & 0.018 & 0.023 & 0.030 & 0.040 & 0.054 & 0.073 & 0.097 & 0.125 & 0.158 & 0.192 & 0.229 & 0.264 & 0.294 \\
\hline $3.136 \ldots \ldots \ldots \ldots$ & 0.012 & 0.015 & 0.018 & 0.024 & 0.032 & 0.044 & 0.060 & 0.081 & 0.106 & 0.137 & 0.170 & 0.206 & 0.242 & 0.275 \\
\hline $3.229 \ldots \ldots \ldots \ldots$ & 0.008 & 0.009 & 0.012 & 0.015 & 0.021 & 0.029 & 0.040 & 0.056 & 0.077 & 0.103 & 0.133 & 0.166 & 0.201 & 0.237 \\
\hline $3.337 \ldots \ldots \ldots \ldots$ & 0.002 & 0.003 & 0.004 & 0.005 & 0.008 & 0.012 & 0.017 & 0.025 & 0.037 & 0.054 & 0.075 & 0.101 & 0.132 & 0.165 \\
\hline $3.461 \ldots \ldots \ldots \ldots$ & -0.002 & -0.002 & -0.002 & -0.001 & 0.000 & 0.002 & 0.004 & 0.008 & 0.014 & 0.023 & 0.035 & 0.052 & 0.074 & 0.101 \\
\hline $3.641 \ldots \ldots \ldots \ldots$ & -0.005 & -0.005 & -0.005 & -0.004 & -0.003 & -0.002 & -0.001 & 0.001 & 0.005 & 0.010 & 0.019 & 0.030 & 0.046 & 0.067 \\
\hline $3.864 \ldots \ldots \ldots \ldots$ & -0.006 & -0.006 & -0.005 & -0.005 & -0.004 & -0.003 & -0.002 & 0.000 & 0.003 & 0.008 & 0.016 & 0.027 & 0.042 & 0.062 \\
\hline $4.118 \ldots \ldots \ldots \ldots$ & -0.006 & -0.006 & -0.005 & -0.005 & -0.004 & -0.003 & -0.001 & 0.001 & 0.005 & 0.011 & 0.020 & 0.032 & 0.049 & 0.071 \\
\hline
\end{tabular}

the grid of model atmospheres are listed in Tables 3 and 4, respectively.

\section{INFERRED C AND N ABUNDANCES AMONG THE SUBGIANTS}

To disentangle the underlying $\mathrm{C}$ and $\mathrm{N}$ abundances from the $\mathrm{CH}$ and $\mathrm{NH}$ band strengths, we have fitted the $[\mathrm{C} / \mathrm{Fe}]$ and $[\mathrm{N} / \mathrm{Fe}]$ abundances corresponding to the observed $I(\mathrm{CH})$ and $I(\mathrm{NH})$ indices of the SGB stars in M15. Since we are using the NH band instead of a $\mathrm{CN}$ band, the coupling between the assumed abundance of $\mathrm{C}$ and the deduced abundance of $\mathrm{N}$ is minimal, and we employ here the same technique of Briley et al. (2002, 2004a; our M13 analysis): the model isoabundance curves of Table 2 were interpolated to the $M_{V}$ of each program star using cubic splines, and the observed index was converted into the corresponding abundance based on the synthetic indices at that $M_{V}$. The resulting $\mathrm{C}$ and $\mathrm{N}$ abundances are plotted in Figure 6 and listed in Table 2 . The error bars were determined by repeating the process while including shifts in the observed indices of twice the average error among the SGB indices as derived from Poisson statistics $[0.005$ in $I(\mathrm{CH})$ and 0.02 in $I(\mathrm{NH})$ ]. The shifts were included in opposing directions [e.g., +0.005 in $I(\mathrm{CH})$ and -0.02 in $I(\mathrm{NH})$ followed by -0.005 in $I(\mathrm{CH})$ and +0.02 in $I(\mathrm{NH})]$ and reflect likely errors in the abundances due to noise in the spectra.

In Figure 3 we show synthetic spectra corresponding to two SGB stars (one NH-strong, the other NH-weak) and a variety of $\mathrm{N}$ abundances. Also shown are the abundances resulting from index matching, which agree well with what one would obtain from visual matching between observed and calculated spectra.

The sensitivity of the derived $\mathrm{C}$ and $\mathrm{N}$ abundances to our assumptions was also evaluated. We chose four representative stars near the base of the RGB and again repeated the fitting of the $\mathrm{CN}$ and $\mathrm{CH}$ band strengths with different values of $[\mathrm{Fe} / \mathrm{H}]$, $[\mathrm{O} / \mathrm{Fe}],{ }^{12} \mathrm{C} /{ }^{13} \mathrm{C}, M_{V}$, and $v_{t}$. These results are presented in Table 5, where it may be seen that the sensitivity of the derived $\mathrm{C}$ and $\mathrm{N}$ abundances to the choice of model parameters is remarkably small (well under 0.2 dex for reasonably chosen values), as would be expected from these weak molecular features. We

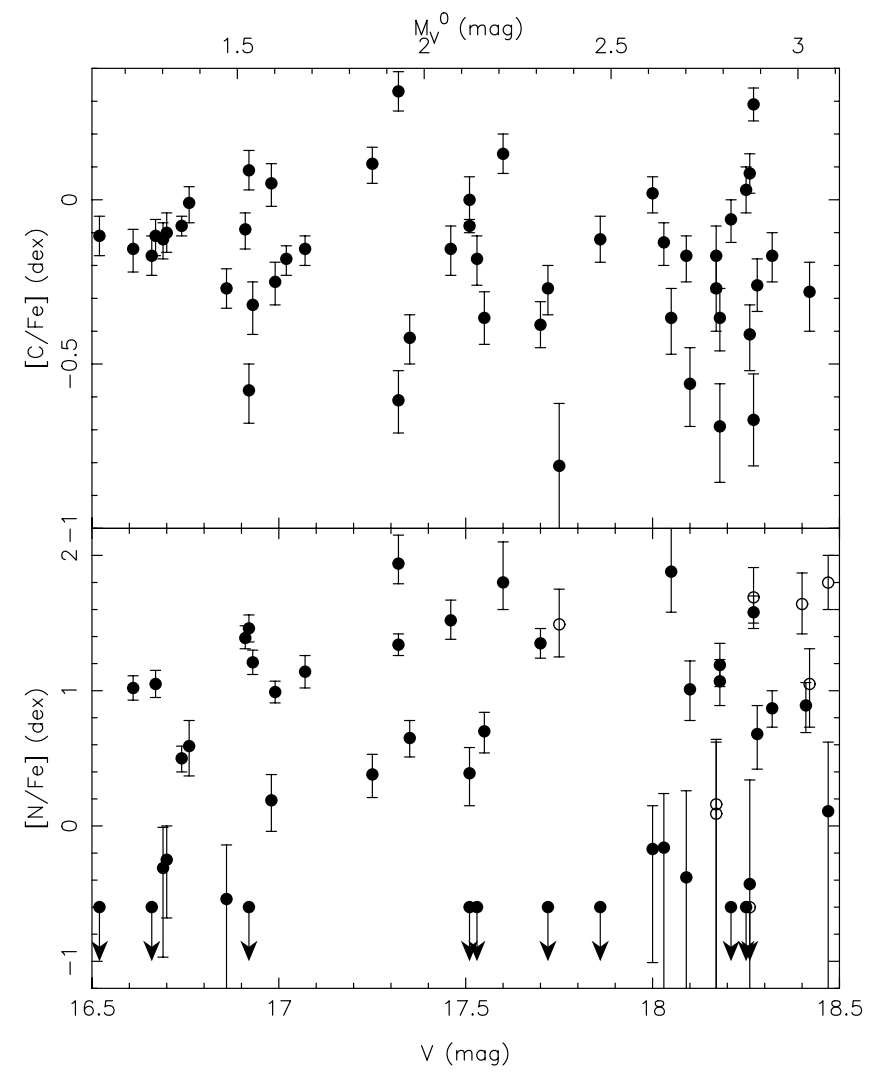

FIG. 6.- $\mathrm{C}$ and $\mathrm{N}$ abundances with respect to Fe for the program stars in M15 as a function of $V$. The top axis is $M_{V}^{0}$. The open circles in [N/Fe] (bottom) denote the stars with the smallest signal level at $3350 \AA$. Large and significant star-to-star differences exist in both $\mathrm{CH}$ and $\mathrm{NH}$ band strengths among these stars. The decreasing spread in indices with luminosity is the result of increasing temperatures near the main-sequence turnoff. Arrows denote upper limits to the $\mathrm{N}$ abundance. 
TABLE 5

Changes in Derived C and N Abundances for Different Model Parameters

\begin{tabular}{|c|c|c|c|c|c|c|c|c|c|c|c|c|}
\hline \multirow[b]{2}{*}{$\mathrm{S}_{\mathrm{TAR}}$} & \multirow[b]{2}{*}[\mathrm{C}/\mathrm{Fe}]{} & \multirow[b]{2}{*}[\mathrm{N}/\mathrm{Fe}]{} & \multicolumn{2}{|c|}{$\Delta(m-M)_{V}=-0.10$} & \multicolumn{2}{|c|}{$\Delta[\mathrm{O} / \mathrm{Fe}]=+0.20$} & \multicolumn{2}{|c|}{${ }^{12} \mathrm{C} /{ }^{13} \mathrm{C}=4^{\mathrm{a}}$} & \multicolumn{2}{|c|}{$[\mathrm{Fe} / \mathrm{H}]=-2.42^{\mathrm{b}}$} & \multicolumn{2}{|c|}{ Turb $=1.5 \mathrm{~km} \mathrm{~s}^{-1}$} \\
\hline & & & $\Delta[\mathrm{C} / \mathrm{Fe}]$ & $\Delta[\mathrm{N} / \mathrm{Fe}]$ & $\Delta[\mathrm{C} / \mathrm{Fe}]$ & $\Delta[\mathrm{N} / \mathrm{Fe}]$ & $\Delta[\mathrm{C} / \mathrm{Fe}]$ & $\Delta[\mathrm{N} / \mathrm{Fe}]$ & $\Delta[\mathrm{C} / \mathrm{Fe}]$ & $\Delta[\mathrm{N} / \mathrm{Fe}]$ & $\Delta[\mathrm{C} / \mathrm{Fe}]$ & $\Delta[\mathrm{N} / \mathrm{Fe}]$ \\
\hline C30215_0807..... & 0.11 & 0.38 & 0.01 & 0.01 & 0.00 & 0.00 & 0.00 & 0.00 & 0.14 & 0.16 & 0.02 & 0.00 \\
\hline C29442_0710..... & -0.61 & 1.34 & 0.01 & 0.01 & -0.01 & 0.00 & 0.00 & 0.00 & 0.23 & 0.15 & 0.04 & 0.01 \\
\hline C29387_0716..... & -0.67 & 1.58 & 0.12 & 0.07 & -0.01 & 0.00 & -0.01 & 0.00 & 0.33 & 0.16 & 0.07 & 0.03 \\
\hline C30146_0829..... & -0.26 & 0.68 & 0.11 & 0.09 & 0.00 & 0.00 & 0.00 & 0.00 & 0.23 & 0.17 & 0.05 & 0.01 \\
\hline
\end{tabular}

${ }^{\text {a }}$ Reduced from the adopted value of ${ }^{12} \mathrm{C} /{ }^{13} \mathrm{C}=10$.

${ }^{b}$ Reduced from the adopted value of $[\mathrm{Fe} / \mathrm{H}]=-2.22 \mathrm{dex}$.

have also plotted the $\mathrm{C}$ and $\mathrm{N}$ abundances of our M15 sample as functions of both $V$ (Fig. 6) and $V-I$ colors (not shown) to evaluate possible systematic effects with luminosity and temperature; none appear to be present.

$\mathrm{C}$ and $\mathrm{N}$ abundances were not calculated for the stars at or below the main-sequence turnoff, as all abundance sensitivity is lost due to the high $T_{\text {eff }}$ and resulting weakness of the molecular bands. For the six stars in our sample with $I(\mathrm{NH}) \leq 1 \%$, essentially all of which have $I(\mathrm{NH})+1 \sigma[I(\mathrm{NH})]>0$, we assign upper limits to $[\mathrm{N} / \mathrm{Fe}]$, which are shown in Figure 6. This figure immediately confirms the very large range in $\mathrm{C}$ and $\mathrm{N}$ abundances from star to star at similar evolutionary stages in M15 previously deduced from the appearance of the $I(\mathrm{CH})-V$ and $I(\mathrm{NH})-V$ plots (Fig. 4).

We next consider whether our data show any correlation between the $\mathrm{C}$ and $\mathrm{N}$ abundances we derive for M15 subgiants and lower RGB stars. Figure 7 shows a plot of $[\mathrm{N} / \mathrm{Fe}]$ versus $[\mathrm{C} / \mathrm{Fe}]$ for the entire M15 sample. An anticorrelation, with considerable scatter, is apparent. The scatter is consistent with the observational errors, but there are a few outliers. In a sample of $70 \mathrm{ob}-$ jects with Gaussian errors, one outlier at the $2.5 \sigma$ level might be expected. One of these, C30123_1138 ( $V=18.27)$, is among the stars with low signal in the continuum near the $\mathrm{NH}$ band, hence $I(\mathrm{NH})$ with a high uncertainty, indicated in Figures 5-9

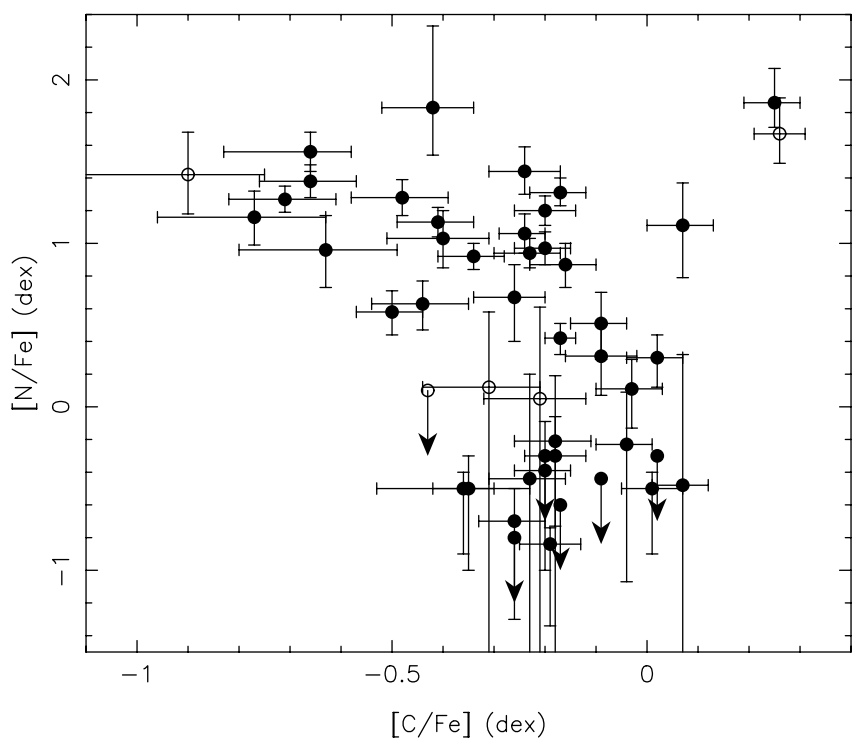

FIG. 7.-Derived [N/Fe] for the M15 stars in Table 2 as a function of the $[\mathrm{C} / \mathrm{Fe}]$ abundances. A C vs. $\mathrm{N}$ anticorrelation is evident. The presence of such an anticorrelation, although suggestive of the presence of atmospheric material exposed to the $\mathrm{CN}$ cycle, is difficult to explain via internal processes given the evolutionary state of the present sample of stars. Arrows denote upper limits to the $\mathrm{N}$ abundance. by open circles. The deviation of star C29413_1023 ( $V=$ 17.32), with extremely enhanced $\mathrm{C}$ and $\mathrm{N}$, from the mean relation shown by the M15 sample in Figure 7 is of higher statistical significance. This star will be discussed in J. B. Cohen \& J. Meléndez (2005, in preparation), in which additional relevant data will be presented. We thus conclude, with the caveat of a very small number of probable outliers, that an anticorrelation between $\mathrm{C}$ and $\mathrm{N}$ is indeed found among the low-luminosity sample of 68 stars in M15 studied here.

Evaluating the accuracy of our absolute abundance scale is more difficult as external comparisons are limited. For the mainsequence stars in 47 Tuc, we can compare the results of Briley et al. (1994), carried out in a manner fairly similar to the present work, with the independent analysis of a different sample of stars by Cannon et al. (1998). This suggests that we may be systematically underestimating the absolute $\mathrm{C}$ abundance by

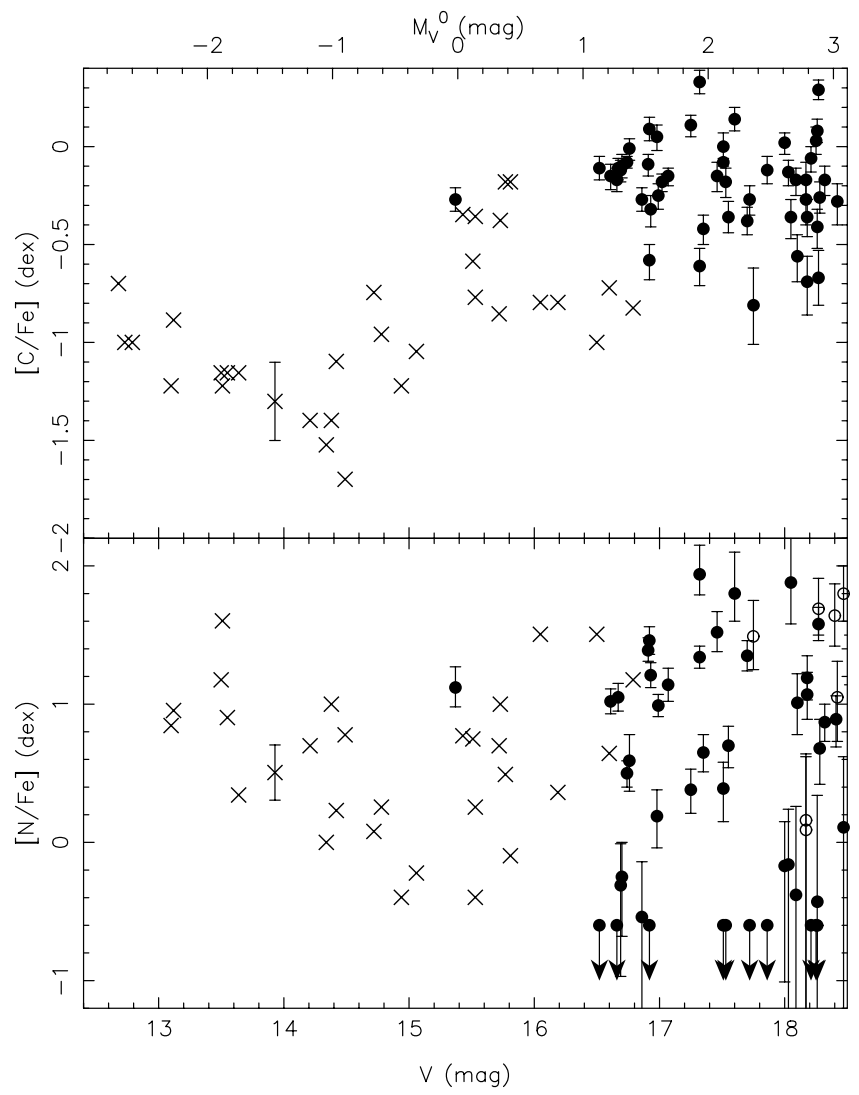

Fig. 8. - Deduced [C/Fe] and [N/Fe] as a function of $V$ mag (with $M_{V}^{0}$ on the top axis) for the low-luminosity stars in M15 from our sample together with the C and N abundances for luminous RGB stars from Trefzger et al. (1983) (crosses). A typical uncertainty for the latter is shown for a single star. 


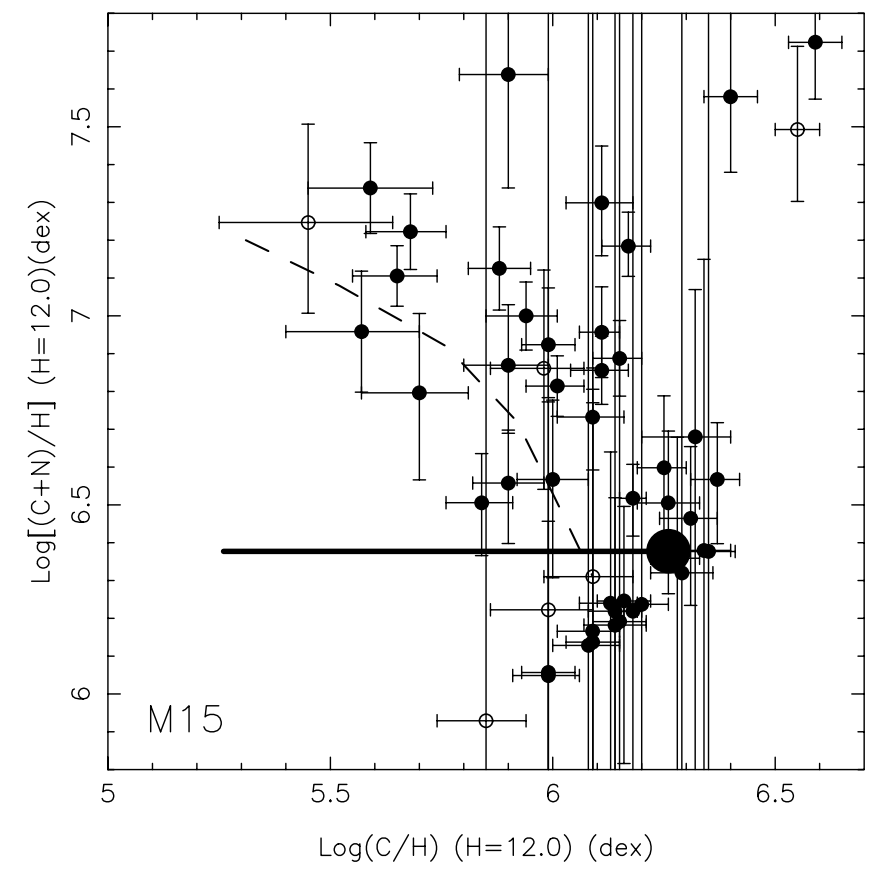

FIG. 9.- Sum of the derived $\mathrm{C}$ and $\mathrm{N}$ abundances as a function of the $\mathrm{C}$ abundance. The large filled circle marks the location for both $\mathrm{C}$ and $\mathrm{N}$ depleted by a factor of 16 , adopting the abundance of $\mathrm{M} 15$ of $[\mathrm{Fe} / \mathrm{H}]=-2.3 \mathrm{dex}$, with $\mathrm{C} / \mathrm{N}$ at the solar ratio. The horizontal line extending to the left represents the locus of points for $\mathrm{C}$ gradually being converted into $\mathrm{N}$, with the left end of the line having $\mathrm{C} / \mathrm{C}_{0}=0.1$. The dashed line indicates the relationship, shown over its full range, that prevails in M5 from our earlier work (Cohen et al. 2002).

about 0.15 dex and overestimating the $\mathrm{N}$ abundance by about 0.2 dex. A second comparison is possible in M13. We obtained $\mathrm{C}$ abundances using our procedures as described here from newly obtained spectra for previously studied bright giants in M13 precisely to address this issue. The mean differences in $[\mathrm{C} / \mathrm{Fe}]$ for our results as compared with literature values was $0.03 \pm$ 0.14 dex for four stars in common with Smith et al. (1996) and $0.14 \pm 0.07$ dex for stars also observed by Suntzeff (1981; if one extreme case is removed) (see Briley et al. 2002 for details). This is very reasonable agreement. It is clear that shifts in the absolute abundance scale cannot account for the large range in $\mathrm{C}$ and $\mathrm{N}$ abundances apparent in Figure 7. We therefore conclude that the $\mathrm{C}$ versus $\mathrm{N}$ anticorrelation among the low-luminosity M15 stars in Figure 7 is indeed real.

\subsection{From the RGB Tip to the Main-Sequence Turnoff}

Trefzger et al. (1983) carried out an extensive analysis of C and $\mathrm{N}$ abundances for the most luminous stars in M15. We now combine our results with theirs, thus sampling the $[\mathrm{C} / \mathrm{Fe}]$ and $[\mathrm{N} / \mathrm{Fe}]$ ratio over the full range of luminosity from the RGB tip to the main-sequence turnoff in M15 in Figure 8.

With the confidence that our absolute abundance scale is reasonably secure and the hope that the same holds for the work of Trefzger et al. (1983), we assert that Figure 8 shows a large range in $[\mathrm{C} / \mathrm{Fe}]$ at low luminosities, accompanied by a decrease in the mean $[\mathrm{C} / \mathrm{Fe}]$ at about $V \sim 15 \mathrm{mag}$, which is essentially the location of the RGB bump in this GC. We take this as evidence of two separate mechanisms contributing to the spread in the abundance of $\mathrm{C}$ and $\mathrm{N}$ in GCs. At high luminosities near the RGB tip, we see evidence of the first dredge-up, as expected from normal stellar evolution, plus the extra mixing common among metal-poor cluster giants, with a decline in the mean $\mathrm{C}$ abundance of about a factor of $5(0.8 \pm 0.3 \mathrm{dex})$; the large uncertainty reflects the possibility that the absolute abundance scale of Trefzger et al. (1983) is different from ours, a matter we plan to investigate in the near future. In metal-poor field giants (Gratton et al. 2000; Spite et al. 2005) a similar drop of about a factor of 2.5 is seen in the $\mathrm{C}$ abundance at about the luminosity of the RGB bump.

These studies offield giants show an increase in $\mathrm{N}$ abundance of about a factor of 4 at $\sim L$ (RGB bump), a drop in the $\mathrm{Li}$ abundance, and a decrease in the ${ }^{12} \mathrm{C} /{ }^{13} \mathrm{C}$ ratio as well. There is some suggestion of an increase in the mean $\mathrm{N}$ abundance for stars in M15 more luminous than $L$ (RGB bump) (Fig. 8), but it is less clear-cut than the drop in mean $[\mathrm{C} / \mathrm{Fe}]$ there. There is also a potential concern of bias, in that Trefzger et al. (1983) could not reliably detect $\mathrm{NH}$ bands weaker than those included here; their paper contains several nondetections that were not plotted in Figure 8.

This is the same phenomenon we identified earlier in M13 (Briley et al. 2002, 2004a), in which the mean $\mathrm{C} / \mathrm{Fe}$ and spread about that value were constant from the subgiants to below the main-sequence turnoff, but stars near the RGB tip showed lower surface $\mathrm{C}$ abundance. In that case, due to the limited data from the literature for the luminous RGB stars, we could not identify the luminosity at which the transition occurred. In M15, as is shown in Figure 8, that transition luminosity is reasonably well defined, and it is $L$ (RGB bump).

\section{ON BURNING}

We next examine whether converting $\mathrm{C}$ to $\mathrm{N}$, presumably via the $\mathrm{CN}$ cycle, is sufficient to reproduce the behavior we have found for our M15 sample or whether burning of the even more abundant element $\mathrm{O}$ is also required. Figure 9 shows the sum of the $\mathrm{C}$ and $\mathrm{N}$ abundance as a function of the $\mathrm{C}$ abundance of the sample of M15 subgiants. The large filled circle shows the predicted location, assuming that the initial $\mathrm{C}$ and $\mathrm{N}$ abundances $\left(\mathrm{C}_{0}, \mathrm{~N}_{0}\right)$ are the solar values reduced by the metallicity of M15 $([\mathrm{Fe} / \mathrm{H}]=-2.3 \mathrm{dex})$. Thus, this is the initial location for no burning and for a solar $\mathrm{C} / \mathrm{N}$ ratio. If the present stars incorporated material in which just $\mathrm{C}$ was burned into $\mathrm{N}$, then the locus of the observed points representing the M15 sample of lowluminosity stars should consist of a single horizontal line, with the initial point, the presence of no $\mathrm{CN}$-cycle exposed material, at the right end of the line (the maximum $\mathrm{C}$ abundance) and the left end of the line corresponding to a substantial fraction of the star's mass (i.e., the atmosphere plus surface convection zone), including C-poor, N-rich material. Furthermore, if the initial $\mathrm{C} / \mathrm{N}$ ratio of the cluster is not solar, then the locus should still be a horizontal line but located at a different vertical height in the figure.

The maximum possible $\mathrm{N}$ enhancement for a cluster SGB star with these assumptions occurs if the star formed entirely from material in which all $\mathrm{C}$ has been converted into N. For initial values $\left(\mathrm{C}_{0}, \mathrm{~N}_{0}\right)$ (not expressed as logarithms), this maximum $\mathrm{N}$ enhancement would be $\left(\mathrm{C}_{0}+\mathrm{N}_{0}\right) / \mathrm{N}_{0}$. If the initial value is the solar ratio, $\mathrm{C}_{0} / \mathrm{N}_{0} \sim 3.2$, the resulting maximum $\mathrm{N}$ enhancement is a factor of $\sim 4.2$, while for an unrealistic initial $\mathrm{C}_{0} / \mathrm{N}_{0}$ of 10 , the maximum $\mathrm{N}$ enhancement is a factor of 11 .

Now we examine the behavior of the $\mathrm{C}$ and $\mathrm{N}$ abundances among the M15 subgiant sample as inferred from our observations. It is clear that the assumption that the only thing happening is inclusion of material in which $\mathrm{C}$ was burned into $\mathrm{N}$ must be incorrect. The sum of $\mathrm{C}+\mathrm{N}$ seems to systematically increase by a factor of $\sim 5$ between the most $\mathrm{C}$-rich star and the most $\mathrm{C}$-deficient star. The discussion of the errors, both internal and systematic, in $\S 6$ suggests maximum systematic errors of 


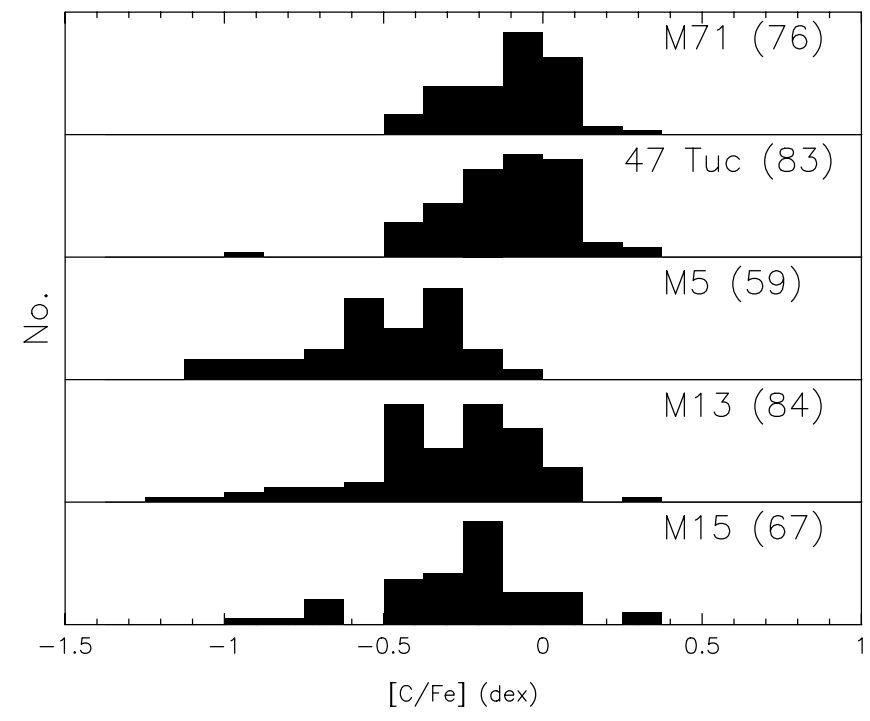

FIG. 10.-Histogram of [C/ Fe] for the low-luminosity stars with suitable data in each of the GCs M15, M13, M5, and M71 from our work (see GC-CN), as well as for 47 Tuc (from Briley et al. 2004b). The region of the main-sequence turnoff is excluded, as the stars there have higher $T_{\text {eff }}$ with very weak molecular bands. The total number of stars included for each cluster is given in parentheses following the cluster name.

-0.2 dex for $\log (\mathrm{C} / \mathrm{H})$ and +0.2 for $\log (\mathrm{N} / \mathrm{H})$. This is completely insufficient to explain such a large trend as a result of systemic errors.

Thus, the sum of $\mathrm{C}+\mathrm{N}$ was not constant as $\mathrm{C}$ was burned into $\mathrm{N}$, wherever that might have occurred. Furthermore, the observed range in $\mathrm{N}$ abundances is very large. The most obvious way to reproduce this is to include $\mathrm{O}$ burning, as well as $\mathrm{C}$ burning. If we adopt solar ratios as our initial values, then a substantial amount of $\mathrm{O}$ burning is required.

Figure 9 suggests that the initial ratio of $\mathrm{C} / \mathrm{N}$ is close to solar. Adopting the solar value as the initial $\mathrm{C} / \mathrm{N}$ ratio, we calculate the minimum amount of $\mathrm{O}$ that must be burned at the base of the AGB envelopes to reproduce the locus observed in the figure (under the arguable assumption of the most extreme of our stars having formed largely from such material; this will, however, provide us with at least an estimate of the minimum burning required). We need to produce a $\mathrm{N}$ enhancement of at least a factor of 10 . The solar ratio is $\mathrm{C} / \mathrm{N} / \mathrm{O}=3.2 / 1 / 7.6$, so if all the $\mathrm{C}$ and $50 \%$ of the $\mathrm{O}$ were converted, we have an enhancement of $\mathrm{N}$ of a factor of 8 available to the present stars. Oxygen is typically found to be overabundant with respect to $\mathrm{Fe}$ in old metal-poor systems (see Meléndez et al. 2001; Gratton et al. 2001; Ramírez \& Cohen 2003; and references therein); we assume $[\mathrm{O} / \mathrm{Fe}] \sim+0.3 \mathrm{dex}$, a typical value. Then the initial $\mathrm{C} / \mathrm{N} / \mathrm{O}$ ratios will be $3.2 / 1 / 15.2$. Note that the same amount of $\mathrm{O}$ has to be burned to produce the observed distribution of $\mathrm{C}$ and $\mathrm{N}$ abundances, but in this case it is a considerably smaller fraction of the initial $\mathrm{O}$.

\section{COMPARISON WITH C AND N STUDIES IN OTHER GLOBULAR CLUSTERS}

We have now analyzed four Galactic GCs covering a wide range in metallicity: M71, M5, M13 (see GC-CN), and the present study of M15. In each case, large samples of stars, all well below the luminosity of the RGB bump, were used. In M13 and M71, we had large samples below the main-sequence turnoff. In this section we attempt to assemble, compare, and inte-

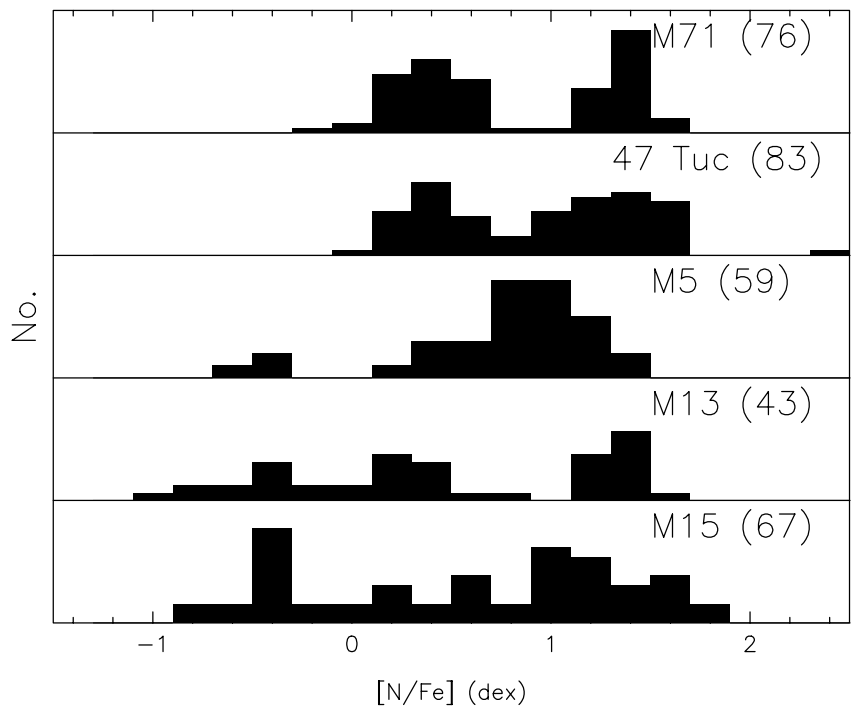

FIG. 11.-Same as Fig. 10 but for [N/Fe].

grate the results of these efforts, adding in other relevant work from the literature. The following section seeks to interpret these results.

The nearby GC 47 Tuc has been studied in great detail by Cannon et al. (1998; see this paper for references to many earlier studies), while Briley et al. (2004b) extend their results by pushing several magnitudes below the main-sequence turnoff of this very nearby cluster. The cluster 47 Tuc and the four clusters we have studied (see GC-CN) are the only GCs for which suitable data exist for the low-luminosity range probed here.

In each of these five GCs, there are large differences in starto-star $\mathrm{C}$ and $\mathrm{N}$ abundances among the low-luminosity stars. Figures 10 and 11 show histograms for the samples of stars in each of these five GCs of the derived $\mathrm{C}$ and $\mathrm{N}$ abundances. From these we estimate the range of variation among these stars for both $\mathrm{C}$ and $\mathrm{N}$ for each cluster, ignoring a few obvious outliers in some cases. The field star $\mathrm{C}$ and $\mathrm{N}$ values for unmixed stars (low-luminosity, metal-poor field giants; presumably these are unmixed stars) from Gratton et al. (2000) $([\mathrm{C} / \mathrm{Fe}] \sim 0.0$, $[\mathrm{N} / \mathrm{Fe}] \sim-0.1 \mathrm{dex})$ roughly coincide with the maximum $\mathrm{C} / \mathrm{Fe}$ ratio and the minimum $\mathrm{N} / \mathrm{Fe}$ ratio.

Our most important new result derives from Figures 10 and 11. These figures clearly show that the range of the spread in both $\mathrm{C}$ and $\mathrm{N}$ is about the same when expressed as $[\mathrm{C} / \mathrm{Fe}]$ and $[\mathrm{N} / \mathrm{Fe}]$ values in each of the five clusters.

An anticorrelation between $\mathrm{C}$ and $\mathrm{N}$ has been found in each of these clusters. This anticorrelation is most easily seen in the metal-rich clusters, as there the observational errors are a smaller fraction of the signal. However, it is seen even in M15. This anticorrelation takes a particular form, illustrated in Figure 9, in which the $[\mathrm{C} / \mathrm{Fe}]-[\mathrm{N} / \mathrm{Fe}]$ relationship we earlier demonstrated to prevail in M5 (dashed curve) is superposed on the results presented here for M15. The length of the curve covers the full range of our M5 stellar data. The agreement of the mean relations we have determined in M5 and M15, both their form and their extent, is very good.

In the metal-rich GCs M71 and 47 Tuc, the $\mathrm{CN}$ and $\mathrm{CH}$ indices appear bimodal, with a preferred high and low value, each varying with luminosity but with few stars occupying the middle ground. However, the more metal-poor GCs, M5, M13, and $\mathrm{M} 15$, show no sign of bimodality for either the $\mathrm{CH}$ or the $\mathrm{CN}$ (or $\mathrm{NH}$ ) line strengths. While an upper limit to band strengths in the 
metal-rich clusters might arise as the bands saturate, the general appearance of the distribution of line indices (see, e.g., Fig. 4 of Cannon et al. 1998) does not support this as an important mechanism here. The bimodality more likely reflects the underlying abundance distributions of $\mathrm{C}$ and $\mathrm{N}$.

By adding in samples of much more luminous stars on the upper giant branch with $\mathrm{C}$ and $\mathrm{N}$ abundances from the literature, we have been able to show that for M13 and M15, the most luminous stars have a mean $\mathrm{C} / \mathrm{Fe}$ ratio about a factor of 3-5 lower than those on the lower giant branch. A similar behavior is present in the large sample of luminous stars in M92 studied by Carbon et al. (1982), in which $\mathrm{C}$ and $\mathrm{N}$ abundances were assigned to 43 giants with $M_{V}<+2$; by Langer et al. (1986); and in the more recent work on $\mathrm{C}$ along the lower RGB of M92 by Bellman et al. (2001). The latter suggest that $\mathrm{C}$ depletion begins at $M_{V}=0.5-1.0 \mathrm{mag}$, while Zoccali et al. (1999) find the RGB bump to be at $M_{V} \sim 0.0 \mathrm{mag}$ in M15 based on HST CMDs. Previous studies of the $\mathrm{C}$ and $\mathrm{N}$ abundances of stars in M5, summarized in Cohen et al. (2002), combined with our low-luminosity large sample in this GC suggest that any difference in the mean $[\mathrm{C} / \mathrm{H}]$ between the tip and the base of the RGB in M5 must be less than 0.3 dex; further observations to refine this are under way. For M71, we can compare our mainsequence $\mathrm{C}$ abundances with those for the $75 \mathrm{RGB}$ giants found by Briley et al. (2001) from David Dunlap Observatory photometry. There is no evidence for any additional $\mathrm{C}$ depletion near the RGB tip in M71, but the uncertainties are large. The situation in 47 Tuc is comical. This GC is so nearby, hence its stars are relatively bright, that large surveys of the luminous giants (see, e.g., Norris et al. 1984) were carried out before the development of molecular band synthesis techniques, and hence they compared $\mathrm{CH}$ and $\mathrm{CN}$ molecular band indices as a function of luminosity but did not derive $\mathrm{C}$ and $\mathrm{N}$ abundances. We have been unable to put together a sample from the literature of stars in 47 Tuc with $\mathrm{C}$ abundances that encompasses the necessary luminosity range in spite of the multitude of published analyses.

This drop in $\mathrm{C} / \mathrm{Fe}$ near the RGB tip is in agreement with the behavior of field stars (Gratton et al. 2000). Thus, as was suggested in Briley et al. (2002), there appear to be two distinct abundance-altering mechanisms that affect GC stars. One produces strong star-to-star scatter at all luminosities, and another, most effective in the metal-poor GCs, produces the drop in $\mathrm{C}$ abundance that starts at $\sim L(\mathrm{RGB}$ bump). Only the latter is seen among field stars, and only the latter is at present understood, by enhanced ("deep") mixing at the first dredge-up once stars evolve to luminosities exceeding $L$ (RGB bump).

\section{IMPLICATIONS FOR STELLAR EVOLUTION}

In the previous section we reviewed the observational results accumulated thus far regarding the $\mathrm{C}$ and $\mathrm{N}$ abundances of stars in Galactic GCs. We note that the sample of well-studied GCs covers a wide range in metallicity and, especially at the metalrich end, a wide range of present cluster mass and central stellar density. In this section we discuss the implications of the collected observational results for stellar evolution of metal-poor GC stars. We find that there appear to be two distinct mixing mechanisms. First, we address the extra $\mathrm{C}$ depletion found on the upper RGB beginning at approximately $L$ (RGB bump). This mechanism is, to first order at least, understood.

A classical review of post-main-sequence stellar evolution can be found in Iben \& Renzini (1983). Their description of the consequences of the first dredge-up phase, the only dredge-up phase to occur prior to the He flash, indicates that a doubling of the surface ${ }^{14} \mathrm{~N}$ and a $30 \%$ reduction in the surface ${ }^{12} \mathrm{C}$ can be expected, together with a drop in the ratio of ${ }^{12} \mathrm{C} /{ }^{13} \mathrm{C}$ from the solar value of 89 to $\sim 20$, as well as a drop in surface Li and B by several orders of magnitude. Observations of metal-poor field stars over a wide range of luminosities conform fairly well to this picture (see, e.g., Shetrone et al. 1993; Gratton et al. 2000), although additional mixing of $\mathrm{Li}$ and lower than predicted ratios of ${ }^{12} \mathrm{C} /{ }^{13} \mathrm{C}$ seem to occur even among field stars (Do Nascimento et al. 2000).

Additional physics was introduced into calculations of dredge-up in old metal-poor stars to better reproduce the observations via "deep mixing." Specific improvements included meridional mixing as described by Sweigart \& Mengel (1979), as well as turbulent diffusion (see Charbonnel 1994, 1995) and the insights of Denissenkov \& Denissenkova (1990) concerning the importance of the ${ }^{22} \mathrm{Ne}(p, \gamma){ }^{23} \mathrm{Na}$ reaction as a way to produce $p$-burning nuclei. The clear prediction of the most recent calculations of this type by Denissenkov \& Weiss (1996), Cavallo et al. (1998), and Weiss et al. (2000) is that the earliest that deep mixing can begin is at the location of the bump in the luminosity function of the RGB that occurs when the H-burning shell crosses a sharp molecular weight discontinuity.

The observations of $\mathrm{C}$ and $\mathrm{N}$ abundances in GC stars (and in field stars; see, e.g., Gratton et al. 2000) are in reasonable agreement with the predictions of the latest such models with regard to the key points: at what luminosity the first dredge-up begins, the amplitude of the decline in $\mathrm{C}$ abundance, and the general shape of the $\mathrm{C}$ depletion as a function of luminosity. The observational situation is not yet adequate to verify the predicted increase of the $\mathrm{N}$ abundance in GC RGB stars above the bump luminosity. The models also predict, in agreement with the observations, that this phenomenon is more efficient at low metallicities, as the thickness of the H-burning shell decreases rapidly and the shell-burning timescale also decreases as the metallicity rises (see Figs. $6 b$ and 7 of Cavallo et al. 1998). The models of Cavallo et al. (1998) and others can predict the strong $\mathrm{O}-\mathrm{Na}$ correlation seen among giants close to the RGB tip in some GCs, particularly the metal-poor ones, as another consequence of $p$ burning in the H-burning shell.

We therefore regard this aspect of the behavior of the $\mathrm{C}$ and $\mathrm{N}$ abundances in GC stars as having a reasonable explanation. It is the very large star-to-star differences in $\mathrm{C}$ and $\mathrm{N}$ abundances found at all luminosities in all GCs studied with sufficient data to date that are not easily explained. The range of variation of $[\mathrm{C} / \mathrm{Fe}]$ and $[\mathrm{N} / \mathrm{Fe}]$ is constant to first order, irrespective of the metallicity of the GC and independent of stellar luminosity, with $\mathrm{C}$ and $\mathrm{N}$ anticorrelated and with maximum $\mathrm{C}$ depletions of a factor of 3-5 accompanying maximum $\mathrm{N}$ enhancement of more than a factor of 10 . The very high $\mathrm{N}$ enhancements require not only $\mathrm{CN}$ burning but $\mathrm{ON}$ burning as well. The range of luminosity over which these $\mathrm{C}$ and $\mathrm{N}$ variations (and also $\mathrm{O}$ and Na variations; see, e.g., Cohen \& Meléndez 2005; Gratton et al. 2001; and references therein) occur in GCs has by now ruled out any scenario that invokes dredge-up and mixing intrinsic to the star itself. We must now regard the fundamental origin of the star-to-star variations in $\mathrm{C}$ and $\mathrm{N}$ abundance we see in GCs as arising outside the stars whose spectra we have studied here.

The strong anticorrelation between $\mathrm{C}$ and $\mathrm{N}$, however, does suggest that $\mathrm{CN}$-cycle material must be involved, and that this material has somehow reached the surface of these lowluminosity GC stars. Since we know it cannot come from inside these stars, it must come from some external source. As reviewed by Lattanzio et al. (2000), CN and ON cycling is known to occur 
in intermediate-mass AGB stars, and such stars are also known to have sufficient dredge-up to bring such material to their surfaces. Recent detailed computations, including both nucleosynthesis with a large set of isotopes and nuclear reaction pathways, have been carried out by Karakas (2003) for very metal-poor intermediate-mass stars. Herwig (2004) has also added in detailed mixing to the stellar surface. These calculations can qualitatively reproduce essentially all of the observational data.

We thus might speculate that the site of the proton exposure could be more rapidly evolving higher mass AGB stars, which then suffer extensive mass loss (either in or outside of binary systems) and pollute the generation of lower mass stars we currently observe, while the higher mass stars are now defunct. Considerable effort to develop this scenario of AGB pollution of the lower mass stars we observe today has been made by Ventura et al. (2001), D'Antona et al. (2002), and, most recently (and most completely), Fenner et al. (2004).

However, the recent observational facts summarized above have, in our view, rendered this scenario not viable either. One problem with any "pollution" scenario is that these abundance inhomogeneities cannot simply be surface contaminations, as they would be diluted by the increasing depth of the convective envelope during RGB ascent. Thus, the amount of accreted mass required to explain the observed $\mathrm{C}$ and $\mathrm{N}$ variations becomes very large. It must be a significant fraction of the total stellar mass, given that a fraction of the star much larger than just the surface convection zone of a luminous RGB star must be contaminated to maintain a constant range of $\mathrm{C}$ and $\mathrm{N}$ at all luminosities. This seems to us unrealistic and contrived, but we note the recent calculations of Thoul et al. (2002), which demonstrate that large accumulations may be possible with some assumptions about stellar orbits, particularly in clusters with small core radii. They estimate that for stars in the core of 47 Tuc, as much as $80 \%$ of the mass of a $1 M_{\odot}$ star in that cluster could be accreted material. Unfortunately, the core radii of some of the GCs we have studied are considerably larger. Even with generous assumptions regarding orbital anisotropy, similar calculations for them yield a much smaller expected accretion $(\sim 10 \%)$, which is not sufficient for present purposes.

Another problem arises because of the tight anticorrelation between $\mathrm{C}$ and $\mathrm{N}$ (see Fig. 9). Any external mechanism for producing these variations will involve an efficiency factor for the incorporation of material. This might be a cross section if accretion from the cluster gas is involved or some property of the accretion disk if binaries are involved. We expect this factor to depend on the mass of the star itself, how much additional mass is incorporated $(\Delta M)$, and the initial $\mathrm{C}$ and $\mathrm{N}$ abundances in the star itself and within $\Delta M$. Since these properties of $\Delta M$ might be expected to fluctuate wildly depending on the mass of the evolved star producing the N-rich ejecta, this process should show a lot of stochastic random variability. Pollution of a lowmass star by ejecta from intermediate-mass AGB stars is just too chaotic and unpredictable to be able to reproduce such wellbehaved trends.

We now turn to the implications of Figures 10 and 11, which display the ranges of $[\mathrm{C} / \mathrm{Fe}]$ and $[\mathrm{N} / \mathrm{Fe}]$ found among lowluminosity stars (stars at the base of the RGB, subgiants, and/or on the main sequence) in five GCs spanning a range in metallicity of a factor of 40. This figure illustrates our most important new result. We see that the maximum and minimum for each of these is approximately the same (to within a factor of 3 ) for each of the clusters. Note that the maxima and minima expressed as $\log [\epsilon(\mathrm{C})]$ and $\log [\epsilon(\mathrm{N})]$ are not constant. Furthermore, the maximum in $[\mathrm{C} / \mathrm{Fe}]$ corresponds reasonably well to that of the
TABLE 6

Comparison of C and N Observed Abundances with Models of Ventura et al. (2002)

\begin{tabular}{|c|c|c|c|c|c|c|}
\hline \multirow[b]{2}{*}[\mathrm{Fe}/\mathrm{H}]{} & \multicolumn{3}{|c|}{ Models } & \multicolumn{3}{|c|}{ OBSERVATIONS } \\
\hline & $\mathrm{C}_{f} / \mathrm{C}_{0}$ & $\mathrm{~N}_{f} / \mathrm{N}_{0}$ & $\begin{array}{c}{\left[\mathrm{N}_{f} / \mathrm{C}_{f}\right]} \\
(\mathrm{dex})\end{array}$ & $\mathrm{C}_{f} / \mathrm{C}_{0}$ & $\mathrm{~N}_{f} / \mathrm{N}_{0}$ & $\begin{array}{c}{\left[\mathrm{N}_{f} / \mathrm{C}_{f}\right]} \\
(\mathrm{dex})\end{array}$ \\
\hline-2.4 (M15) .................... & 0.75 & 28 & +1.6 & 0.20 & 100 & +2.7 \\
\hline-0.8 (M71, 47 Tuc)..... & 0.11 & 7 & +1.8 & 0.25 & 15 & +1.8 \\
\hline
\end{tabular}

field stars, while the minimum in [N/Fe] corresponds to that of the field stars.

Thus, it seems reasonable that, as is commonly assumed, the high- $\mathrm{C}$, low-N stars represent the nominal chemical inventory, while the abnormal ones are those with low $\mathrm{C}$ and high $\mathrm{N}$. The isotope ${ }^{12} \mathrm{C}$ is produced by the triple- $\alpha$ process and destroyed by CN burning, while ${ }^{14} \mathrm{~N}$ is produced via $\mathrm{CN}$ and "hot bottom" burning. We therefore expect $\mathrm{N}$ to behave as a primary element, while the behavior of $\mathrm{C}$ may be more complex. The observations, however, demand that the additional material dumped onto the low-C, high-N stars is not from some primary process in which a fixed amount of $\mathrm{N}$ per gram is produced, dispersed into the $\mathrm{GC}$, and mixed into the GC gas. Instead, the chemical inventory of $\mathrm{C}$ and $\mathrm{N}$ behaves like a secondary process, increasing as $[\mathrm{Fe} / \mathrm{H}]$ increases.

The modeling of the production and dredge-up of elements such as $\mathrm{C}$ and $\mathrm{N}$ in AGB stars, while still very uncertain, is rapidly advancing. Detailed models, such as those of Karakas (2003) of yields and abundances for various species at the surfaces of such stars after dredge-up, are now available. So we must ask what the surface ratios are of the species of interest in intermediate-mass AGB stars after dredge-up and what the relevant mass-loss rates, which will drive the processed material into the cluster gas, are. Mass loss in AGB stars is primarily driven by radiation pressure on dust grains (Vasiliadis \& Wood 1993). While there is still some uncertainty, recent comparisons of heavily obscured AGB stars in the SMC, the LMC, and the Milky Way by van Loon (2000) suggest that the total mass-loss rate for a star of fixed luminosity is only weakly dependent on metallicity.

Chemical yields for intermediate-mass stars have been given by Marigo (2001), Ventura et al. (2002), and Karakas (2003). We examine the ratio of initial to final $\mathrm{C}$ and $\mathrm{N}$ in models of different initial metallicity to determine how closely the behavior of ${ }^{12} \mathrm{C}$ and ${ }^{14} \mathrm{~N}$ in the ejecta of such stars matches the extreme range of the observations in GCs over a wide range in metallicity. Table 6 presents this comparison in detail for the models of Ventura et al. (2002); those of Karakas (2003) and Gavilan et al. (2005) do not cover a sufficient range in metallicity to be useful for our purpose, while the results of Marigo (2001) were not given in tabular form.

We find that, while correct in sign, the models of Ventura et al. (2002) fail to reproduce the observations by factors of up to $\sim 10$, depending on which of the three ratios presented in the table is examined. The worst discrepancy is in the final ratio $\mathrm{C} / \mathrm{N}$ after dredge-up in the most metal-poor GCs. There the extent of both the depletion of $\mathrm{C}$ and the enhancement of $\mathrm{N}$ are badly underestimated by the models. Fenner et al. (2004) also find that ejecta from intermediate-mass AGB stars cannot reproduce details of the abundance distributions of the $\mathrm{Mg}$ isotope ratios in NGC 6752 (see also Denissenkov \& Herwig 2003). AGB ejecta can be observed directly by studying them in situ, i.e., in planetary 
nebulae. An independent verification comparison for the predicted yields can be attempted via abundance analyses of the planetary nebulae in the SMC. Stranghellini et al. (2005) discuss the planetary nebulae in the LMC, whose metallicity is higher than that of any GC considered here. The SMC planetary nebulae are not as well studied, and with the demise of the Space Telescope Imaging Spectrograph on the $H S T$, future progress in this area will be, at best, very slow.

Although the predictions of Ventura et al. (2002) and Karakas (2003) for the behavior of C and $\mathrm{N}$ after dredge-up of intermediatemass AGB stars are reasonably consistent with each other, the uncertainty in these calculations must be large. Whether it is large enough to accommodate discrepancies of a factor of $\sim 10$ in $\mathrm{C} / \mathrm{N}$ ratios is not clear. An optimist would say that this level of (dis)agreement is satisfactory, given the difficulties and complexity of the modeling effort required, while a pessimist would say that these discrepancies are larger than can be reasonably expected from the models and data. A very recent paper, Ventura \& D'Antona (2005), discusses the modeling uncertainties arising from just one issue: the description of convection adopted. They find that changes of a factor of 2 in predicted $\mathrm{C}$ and $\mathrm{N}$ surface abundances in intermediate-mass metal-poor AGB stars are easily achieved by this means. We choose to be optimists. More such modeling efforts, even though they require many assumptions, will be very valuable.

\section{IMPLICATIONS FOR GLOBULAR CLUSTER FORMATION AND CHEMICAL EVOLUTION}

In addition to the accumulated evidence regarding $\mathrm{C}$ and $\mathrm{N}$ abundances presented above, there is one other key fact that must figure in any model of the chemical evolution of GCs. This is that the abundances of the heavy elements, particularly those between $\mathrm{Ca}$ and the Fe peak, are constant for all stars in a GC. Extensive efforts (see, e.g., Cohen \& Meléndez 2005) have failed to detect any dispersion larger than the observational errors. The abundance spreads are confined to the light elements. ${ }^{7}$

We suggest that a viable scenario for the chemical evolution of GCs can only be constructed if GC stars are not all coeval, and more than one epoch of star formation in GCs must have occurred, albeit all within a relatively short timescale. During the early stages, a variation in $\mathrm{C}$ and $\mathrm{N}$ abundances satisfying the above observational data was imprinted on the protocluster gas before the present generation of stars we now observe was formed. The low-mass stars we currently observe formed from the polluted gas some time later during the extended period of star formation in GCs. Furthermore, if one is a pessimist, one must rule out some previous-generation intermediate-mass AGB stars as the source of this pollution because of problems in the predicted $\mathrm{C} / \mathrm{N}$ ratios. If one is an optimist, then one ascribes these problems to modeling difficulties, to mass-loss rates increasing dramatically with increasing metallicity, which is not supported by observations (van Loon 2000), or to some other such factor, and assumes that these AGB stars did generate the $\mathrm{C}$ and $\mathrm{N}$ variations seen today in low-luminosity GC stars.

A tentative scenario that fits most of the facts might be that the first stars to form in the protocluster gas were very massive. Since this gas might have had very low metallicity, theoretical support for an initial mass function heavily biased toward highmass stars under these conditions can be found in the review of Bromm \& Larson (2004). The Type II supernovae (SNe II) from these stars produced the heavy elements through the $\mathrm{Fe}$

\footnotetext{
7 There is increasing evidence that there may be some variation of the heavy and very rare $r$ - and $s$-process elements in GCs. We ignore this here.
}

peak seen in GC stars. The violent explosions ejected energy into the cluster gas, which kept it well mixed. This is crucial to maintaining constant abundances of the heavy elements in the stars within a particular GC. SN II explosions may also have acted to disrupt the lowest mass protoclusters, which became halo field stars.

The lifetimes of high-mass stars are very short, and so was the duration of this phase of evolution of the GC. After some (short) time, no more massive stars were formed. Intermediatemass stars began to form with metallicity the same as that of the GC as seen today. Such stars have typical lifetimes of $\sim 2$ Gyr. During the course of their evolution, they produced material in their interiors that went through the $\mathrm{CN}$ process (and the $\mathrm{ON}$ process, to some extent). This material was subsequently ejected, but the gas was no longer mixed globally over the cluster volume, and local pockets of substantial or negligible enrichment of the light elements developed. Since GC CMDs do not permit an age range of 2 Gyr among the low-mass GC stars, no low-mass stars could have formed until near the end of this second phase. At this point, the low-mass stars that we see today formed with variable light-element ratios but fixed heavy-element abundances.

It is now possible to include the formation of GCs in cosmological simulation (Kravtsov \& Gnedin 2005). However, the level of detail needed here to follow their chemical evolution with regard to the light elements is still beyond our capabilities. Although the overall picture sketched above seems reasonable, current models for nucleosynthesis and dredge-up for intermediate-mass AGB stars fail to reproduce in detail the observed $\mathrm{C}$ and $\mathrm{N}$ variations in GC stars, in the sense that the $\mathrm{C}$ depletions and $\mathrm{N}$ enhancements observed in low-metallicity GCs are considerably larger than theory predicts. Unless those models are flawed, the relatively short-lived stellar source for the second phase of this scenario, when the cluster gas is no longer well mixed throughout its volume, is unknown.

In evaluating such a scenario, it is important to remember that the present mass of a GC may be much lower than its initial mass as a protocluster; stars are lost from the cluster through many processes (see, e.g., Mashchenko \& Sills 2005). Thus, the absence of a relation between the present mass (or central density) of a $\mathrm{GC}$ and its $[\mathrm{Fe} / \mathrm{H}]$ should not be surprising. It would be of interest to test to even greater accuracy the constancy of the Fe peak elements within a particular GC.

\section{SUMMARY}

We present moderate-resolution spectroscopy and photometry for a large sample of subgiants and stars at the base of the RGB in the extremely metal-poor Galactic globular cluster M15 (NGC 7078), with the goal of deriving $\mathrm{C}$ abundances (from the $\mathrm{G}$ band of $\mathrm{CH}$ ) and $\mathrm{N}$ abundances (from the NH band at $3360 \AA$ ). Star-to-star stochastic variations with significant range in $[\mathrm{C} / \mathrm{Fe}]$ and especially $[\mathrm{N} / \mathrm{Fe}]$ are found at all luminosities extending to the subgiants at $M_{V} \sim+3$.

An analysis of these Keck LRIS spectra with theoretical synthetic spectra reveals that these star-to-star variations between $\mathrm{C}$ and $\mathrm{N}$ abundances are anticorrelated, as would be expected from the presence of proton-capture-exposed material in our sample stars. The evolutionary states of these stars are such that the currently proposed mechanisms for in situ modifications of $\mathrm{C}, \mathrm{N}$, and $\mathrm{O}$ have yet to take place. On this basis, we infer that the source of proton exposure lies not within the present stars but more likely in a population of more massive stars that have "polluted" our sample.

The range of variation of the $\mathrm{N}$ abundances is very large, and the sum of $\mathrm{C}+\mathrm{N}$ increases as $\mathrm{C}$ decreases. To reproduce this 
requires the incorporation not only of $\mathrm{CN}$ but also of $\mathrm{ON}$ processed material, as we also found earlier for M5 (see GC-CN).

We combine our work with that of Trefzger et al. (1983) for the brighter giants in M15 to extend coverage to a larger luminosity range reaching from the RGB tip to the main-sequence turnoff. We then find strong evidence for additional depletion of $\mathrm{C}$ among the most luminous giants. This presumably represents the first dredge-up (with enhanced deep mixing) expected for such luminous RGB stars in the course of normal stellar evolution as they cross the RGB bump.

Our work now covers four GCs (M15, M13, M5, and M71; see GC-CN) spanning a metallicity range of a factor of 40 . We look at the trends of $\mathrm{C}$ and $\mathrm{N}$ abundances common to all the GCs studied to date, including (from the literature) 47 Tuc. While all clusters studied show strong anticorrelated variations of $\mathrm{C}$ and $\mathrm{N}$ at all luminosities probed, the metal-rich clusters (M71, 47 Tuc, and M5) do not show evidence for the first dredge-up among their most luminous giants, while the metal-poor ones (M13, M92, and M15, plus M5) do. This is predicted by the models of the first dredge-up on the RGB, which can reproduce essentially all the key features of the associated changes in $\mathrm{C}$ abundance, including the luminosity at which it begins and the amplitude of the decline in $[\mathrm{C} / \mathrm{Fe}]$ as a function of metallicity. The metal-poor clusters do not show evidence for the bimodality in $\mathrm{CH}$ and $\mathrm{CN}$ line strengths seen in the metal-rich clusters. The origin of the bimodality is unclear.

It is the star-to-star variations in $\mathrm{C}$ and $\mathrm{N}$ seen at low luminosity in all these GCs that is more difficult to explain. Having eliminated in situ CN processing, pollution by material from intermediate-mass AGB stars is the most popular current scenario to produce this. However, we rule out this suggestion, at least as far as accretion onto existing stars is concerned.

Our most important new result is that the range of $[\mathrm{C} / \mathrm{Fe}]$ and [N/Fe] seen in these five GCs is approximately constant (see Figs. 10 and 11), i.e., $\mathrm{C}$ and $\mathrm{N}$ are behaving as though they were produced via a secondary, not a primary, nucleosynthesis process. A detailed comparison of our results with the models for nucleosynthesis and dredge-up of low-metallicity intermediatemass AGB stars by Ventura et al. (2002) fails to explain the details of the $\mathrm{C}$ and $\mathrm{N}$ abundances, predicting key ratios incorrectly by up to a factor of $\sim 10$. Thus, pollution of cluster gas by such stars can also be ruled out unless current models of surface $\mathrm{N}$ abundances after dredge-up are flawed, which seems possible given the complexity of the modeling and the many assumptions required.

The behavior of the $\mathrm{C}$ and $\mathrm{N}$ abundances among lowluminosity stars in $\mathrm{GCs}$, while $[\mathrm{Fe} / \mathrm{H}]$ is constant to high precision within each $\mathrm{GC}$, forces us to assume that there was an extended period of star formation in GCs. The first stars were exclusively of high mass, and their SN II ejecta produced the heavy metals seen in the GC. A second generation of short-lived stars of an unknown type (not intermediate-mass AGB stars, unless current models are flawed) evolved, ejected mass, and polluted the GC gas with light elements; the low-mass stars we see today formed afterward.

The entire Keck HIRES and LRIS user communities owe a huge debt to Jerry Nelson, Gerry Smith, Steve Vogt, Bev Oke, and many other people who have worked to make the Keck Telescope and HIRES and LRIS a reality and to operate and maintain the Keck Observatory. We are grateful to the W. M. Keck Foundation for the vision to fund the construction of the W. M. Keck Observatory. The authors wish to extend special thanks to those of Hawaiian ancestry on whose sacred mountain we are privileged to be guests. Without their generous hospitality, none of the observations presented herein would have been possible.

J. G. C. acknowledges support from the National Science Foundation (under grant AST 02-5951), and M. M. B. acknowledges support from the National Science Foundation (under grant AST 00-98489) and the F. John Barlow endowed professorship. We are also in debt to Roger Bell for the use of the SSG program, the dean of the University of WisconsinOshkosh College of Letters and Sciences for the workstation that made the extensive modeling possible, and Jorge Meléndez for the IR observations.

This work has made use of the USNOFS Image and Catalog Archive operated by the United States Naval Observatory, Flagstaff Station (http://www.nofs.navy.mil/data/fchpix). This publication makes use of data from the Two Micron All Sky Survey, which is a joint project of the University of Massachusetts and the Infrared Processing and Analysis Center, funded by the National Aeronautics and Space Administration and the National Science Foundation.

\section{REFERENCES}

Asplund, M., Grevesse, N., \& Sauval, A. J. 2005, in ASP Conf. Ser. 336, Cosmic Abundances as Records of Stellar Evolution and Nucleosynthesis, ed. F. N. Bash \& T. G. Barnes (San Francisco: ASP), in press

Bell, R. A., \& Gustafsson, B. 1978, A\&AS, 34, 229 1989, MNRAS, 236, 653

Bell, R. A., Paltoglou, G., \& Tripicco, M. J. 1994, MNRAS, 268, 771

Bellman, S., Briley, M. M., Smith, G. H., \& Claver, C. F. 2001, PASP, 113, 326

Briley, M. M., \& Cohen, J. G. 2001, AJ, 122, 242

Briley, M. M., Cohen, J. G., \& Stetson, P. B. 2002, ApJ, 579, L17 2004a, AJ, 127, 1579

Briley, M. M., Harbeck, D., Smith G. H., \& Grebel, E. K. 2004b, AJ, 127, 1588

Briley, M. M., Hesser, J. E., Bell, R. A., Bolte, M., \& Smith, G. H. 1994, AJ, 108,2183

Briley, M. M., \& Smith, G. H. 1993, PASP, 105, 1260

Briley, M. M., Smith, G. H., \& Claver, C. F. 2001, AJ, 122, 2561

Bromm, V., \& Larson, R. B. 2004, ARA\&A, 42, 79

Buonanno, R., Corsi, C. E., \& Fusi Pecci, F. 1985, A\&A, 145, 97

Cannon, R. D., Croke, B. F. W., Bell, R. A., Hesser, J. E., \& Stathakis, R. A. 1998, MNRAS, 298, 601

Carbon, D. F., Langer, G. E., Butler, D., Kraft, R., Suntzeff, N., Kemper, E., Trefzger, C., \& Romanishin, W. 1982, ApJS, 49, 207

Cavallo, R. M., Sweigart, A. V., \& Bell, R. A. 1998, ApJ, 492, 575

Charbonnel, C. 1994, A\&A, 282, 811

1995, ApJ, 453, L41

Cohen, J. G. 1999, AJ, 117, 2434

Cohen, J. G., Briley, M. M., \& Stetson, P. B. 2002, AJ, 123, 2525

Cohen, J. G., \& Meléndez, J. 2005, AJ, 129, 303

Cutri, R. M., et al. 2003, Explanatory Supplement to the 2MASS All-Sky Data Release (Pasadena: IPAC), http://www.ipac.caltech.edu/2mass/releases/allsky/ doc/explsup.html

DaCosta, G. S., \& Armandroff, T. E. 1990, AJ, 100, 162

D’Antona, F., Caloi, V., Montalban, J., Ventura, P., \& Gratton, R. 2002, A\&A, 395,69

Demarque, P., Woo, J., Kim, Y., \& Yi, S. K. 2004, ApJS, 155, 667

Denissenkov, P. A., \& Denissenkova, S. N. 1990, Soviet Astron. Lett., 16, 275

Denissenkov, P. A., \& Herwig, F. 2003, ApJ, 590, L99

Denissenkov, P. A., \& Weiss, A. 1996, A\&A, 308, 773

Do Nascimento, J. D., Jr., Charbonnel, C., Lèbre, A., de Laverny, P., \& De Medeiros, J. R. 2000, A\&A, 357, 931

Durrell, P. B., \& Harris, W. E. 1993, AJ, 105, 1420

Fenner, Y., Campbell, S., Karakas, A. I., Lattanzio, J. C., \& Gibson, B. K. 2004, MNRAS, 353, 789

Gavilan, M., Buell, J. F., \& Molla, M. 2005, A\&A, 432, 861

Gratton, R. G., Sneden, C., \& Carretta, E. 2004, ARA\&A, 42, 385 
Gratton, R. G., Sneden, C., Carretta, E., \& Bragaglia, A. 2000, A\&A, 354, 169

Gratton, R. G., et al. 2001, A\&A, 369, 87

Gustafsson, B., \& Bell, R. A. 1979, A\&A, 74, 313

Gustafsson, B., Bell, R. A., Eriksson, K., \& Nordlund, Å. 1975, A\&A, 42, 407

Harris, W. E. 1996, AJ, 112, 1487

Herwig, F. 2004, ApJS, 155, 651

Hesser, J. E. 1978, ApJ, 223, L117

Iben, I., Jr., \& Renzini, A. 1983, ARA\&A, 21, 271

Karakas, A. I. 2003, Ph.D. thesis, Monash Univ.

Kraft, R. P. 1994, PASP, 106, 553

Kravtsov, A. V., \& Gnedin, O. Y. 2005, ApJ, 623, 650

Landolt, A. R. 1992, AJ, 104, 340

Langer, G. E. 1985, PASP, 97, 382

Langer, G. E., Kraft, R. P., Carbon, D. F., Friel, E., \& Oke, J. B. 1986, PASP, 98, 473

Lattanzio, J., Forestini, M., \& Charbonnel, C. 2000, Mem. Soc. Astron. Italiana, 71,737

Marigo, P. 2001, A\&A, 370, 194

Mashchenko, S., \& Sills, A. 2005, ApJ, 619, 243

McCarthy, J. K., et al. 1998, Proc. SPIE, 3355, 81

Meléndez, J., Barbuy, B., \& Spite, F. 2001, ApJ, 556, 858

Monet, D. G., et al. 1998, USNO-A2.0 Catalog (Flagstaff: USNO)

Norris, J. E., Freeman, K. C., \& DaCosta, G. S. 1984, ApJ, 277, 615

Oke, J. B., et al. 1995, PASP, 107, 375

Pinsonneault, M. 1997, ARA\&A, 35, 557

Ramírez, S. V., \& Cohen, J. G. 2002, AJ, 123, 3277 2003, AJ, 125, 224

Recio-Blanco, A., et al. 2005, A\&A, 432, 851

Shetrone, M. D., Sneden, C., \& Pilachowski, C. A. 1993, PASP, 105, 337

Shortridge, K. 1993, in ASP Conf. Ser. 52, Astronomical Data Analysis Software and Systems II, ed. R. J. Hannisch, R. J. V. Brissenden, \& J. Barnes (San Francisco: ASP), 219
Skrutskie, M. F., et al. 1997, in The Impact of Large Scale Near-IR Sky Surveys, ed. F. Garzon et al. (Dordrecht: Kluwer), 25

Smith, G. H., Shetrone, M. D., Bell, R. A., Churchill, C. W., \& Briley, M. M. 1996, AJ, 112, 1511

Sneden, C., Kraft, R. P., Shetrone, M. D., Smith, G. H., Langer, G. E., \& Prosser, C. 1997, AJ, 114, 1964

Spite, M., et al. 2005, A\&A, 430, 655

Stetson, P. B. 1987, PASP, 99, 191 2000, PASP, 112, 925

Stranghellini, L., Shaw, R. A., \& Gilmore, D. 2005, ApJ, 622, 294

Suntzeff, N. B. 1981, ApJS, 47, 1

Sweigart, A. V., \& Mengel, J. G. 1979, ApJ, 229, 624

Thoul, A., Jorissen, A., Goriely, S., Jehin, E., Magain, P., Noels, A., \& Parmentier, G. 2002, A\&A, 383, 491

Trefzger, C. F., Carbon, D. F., Langer, G. E., Suntzeff, N. B., \& Kraft, R. P. 1983, ApJ, 266, 144

Tripicco, M. J., \& Bell, R. A. 1995, AJ, 110, 3035

van Loon, Th. 2000, A\&A, 354, 125

Vasiliadis, E., \& Wood, P. R. 1993, ApJ, 413, 641

Ventura, P., \& D’Antona, F. 2005, A\&A, 431, 279

Ventura, P., D’Antona, F., \& Mazzitelli, I. 2002, A\&A, 393, 215

Ventura, P., D’Antona, F., Mazzitelli, I., \& Gratton, R. 2001, ApJ, 550, L65

Weiss, A., Denissenkov, P. A., \& Charbonnel, C. 2000, A\&A, 356, 181

Wilson, W. L., et al. 2003, Proc. SPIE, 5216, 178

Yi, S., Demarque, P., Kim, Y.-C., Lee, Y.-W., Ree, C., Lejeune, Th., \& Barnes, S. 2001, ApJS, 136, 417

Zoccali, M., Cassisi, G., Piotto, G., Bono, G., \& Salaris, M. 1999, ApJ, 518, L49 